\title{
Article
}

\section{Design and Control of an Inflatable Spherical Robotic Arm for Pick and Place Applications}

\author{
Matthias Hofer*(D), Jasan Zughaibi and Raffaello D'Andrea \\ Institute for Dynamic Systems and Control, ETH Zurich, Sonneggstrasse 3, 8092 Zurich, Switzerland; \\ zjasan@student.ethz.ch (J.Z.); rdandrea@ethz.ch (R.D.) \\ * Correspondence: hofermat@ethz.ch
}

Citation: Hofer, M.; Zughaibi, J.; D'Andrea, R. Design and Control of an Inflatable Spherical Robotic Arm for Pick and Place Applications. Actuators 2021, 10, 299. https:// doi.org/10.3390/act10110299

Academic Editor: Alessio Merola

Received: 7 October 2021

Accepted: 5 November 2021

Published: 11 November 2021

Publisher's Note: MDPI stays neutral with regard to jurisdictional claims in published maps and institutional affiliations.

Copyright: (c) 2021 by the authors. Licensee MDPI, Basel, Switzerland. This article is an open access article distributed under the terms and conditions of the Creative Commons Attribution (CC BY) license (https:/ / creativecommons.org/licenses/by/ $4.0 /)$.

\begin{abstract}
We present an inflatable soft robotic arm made of fabric that leverages state-of-the-art manufacturing techniques, leading to a robust and reliable manipulator. Three bellow-type actuators are used to control two rotational degrees of freedom, as well as the joint stiffness that is coupled to a longitudinal elongation of the movable link used to grasp objects. The design is motivated by a safety analysis based on first principles. It shows that the interaction forces during an unexpected collision are primarily caused by the attached payload mass, but can be reduced by a lightweight design of the robot arm. A control allocation strategy is employed that simplifies the modeling and control of the robot arm and we show that a particular property of the allocation strategy ensures equal usage of the actuators and valves. The modeling and control approach systematically incorporates the effect of changing joint stiffness and the presence of a payload mass. An investigation of the valve flow capacity reveals that a proper timescale separation between the pressure and arm dynamics is only given for sufficient flow capacity. Otherwise, the applied cascaded control approach can introduce oscillatory behavior, degrading the overall control performance. A closed form feed forward strategy is derived that compensates errors induced by the longitudinal elongation of the movable link and allows the realization of different object manipulation applications. In one of the applications, the robot arm hands an object over to a human, emphasizing the safety aspect of the soft robotic system. Thereby, the intrinsic compliance of the robot arm is leveraged to detect the time when the robot should release the object.
\end{abstract}

Keywords: soft robotics; fabric bellows; pneumatic actuation; control allocation; linear parameter varying model; timescale separation; cascaded control; stiffness control; pick and place application

\section{Introduction}

Soft robotic manipulators combine a number of properties that make them interesting for close collaboration with humans: They are lightweight and compliant, which reduces the risk of injury for humans in the case of an unexpected collision. We present an inflatable robot arm for object manipulation made from fabric and actuated by three bellow-type actuators (see Figure 1). The system has two rotational degrees of freedom and is able to adjust the joint stiffness that is coupled to a longitudinal elongation of the movable part, which proves useful for grasping objects. The modeling approach captures the fundamental dynamics of the system that are strongly dependent on the adjustable joint stiffness and a possible payload mass attached to the tip of the system. Both effects are incorporated in the parametric model and leveraged by the control approach. An important property of the control allocation strategy is investigated, and its connection to the available valve flow capacity in turn has strong implications for the timescale separation that forms the basis of the cascaded control architecture. 


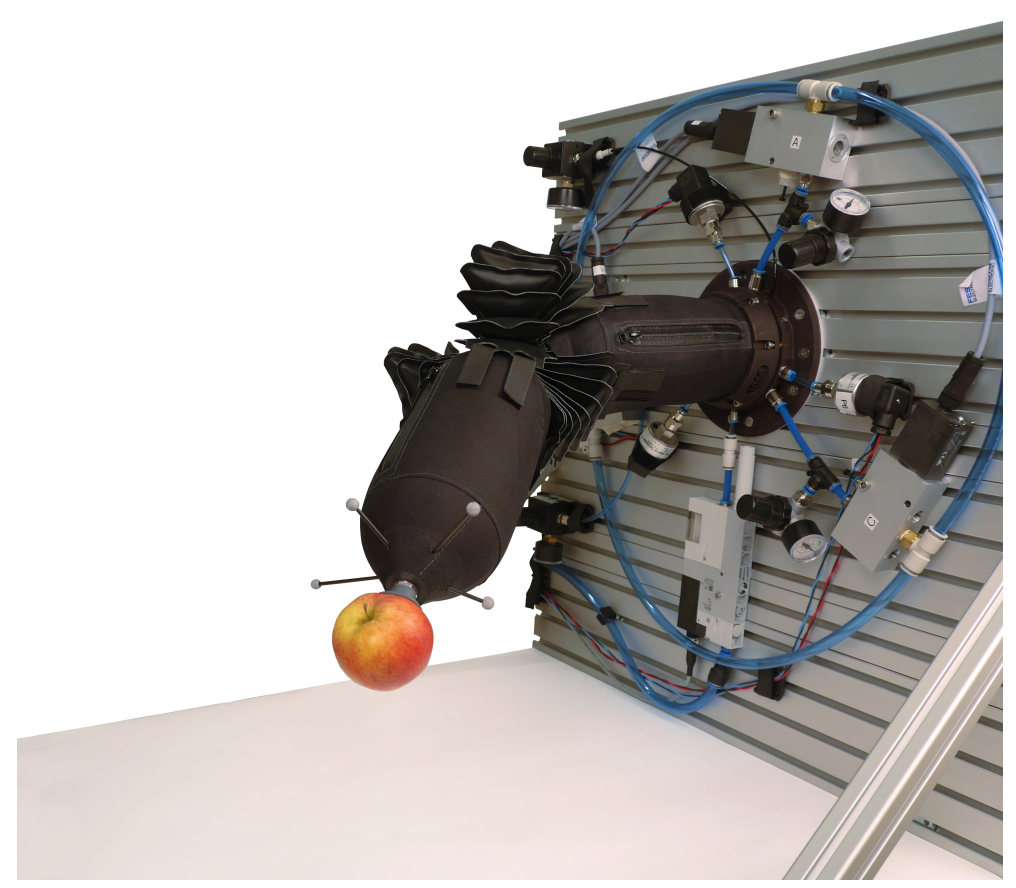

Figure 1. The inflatable soft robotic arm presented in this work shown manipulating an apple. The system is made of fabric and uses three bellow-type actuators to control its two rotational degrees of freedom and adjust its joint stiffness.

\subsection{Related Work}

Ensuring the safety of humans that are in close contact with robotic manipulators has been actively researched for rigid robots for decades. The problem can be addressed by the emulation of compliance by the feedback controller, referred to as impedance control [1,2], or by introducing compliant components into a rigid manipulator [3]. Alternatively, the whole robot manipulator can be built from compliant materials, resulting in a soft robot $[4,5]$. The safety of soft robots has already been studied in earlier work [6] and more recently has been investigated and formalized in [7].

The design space of soft robots is significant and includes numerous materials, actuation principles, and fabrication techniques. A general summary of the field is given in [8] and an overview of pneumatically actuated soft robots is provided in $[9,10]$. Air-driven actuators can be divided into three main groups: The first group of systems contracts upon pressurization. Examples of this are pneumatic artificial muscles (PAM) [11] or actuators that expand in one direction, causing a contraction in another direction [12]. The second actuation principle uses negative pressure (i.e., vacuum) to cause a contraction of the actuator. The third group of actuators expands upon pressurization, with the bellow-type actuator being an example (see [13] for an overview), as well as the so-called pouch motors [14].

The fabrication of air-driven soft robots depends on the material employed and is typically done by either casting [15], 3D printing [16], or a layered manufacturing process using sewing [17]; thermal bonding by means of a heat press [18], or high frequency welding [19]. Finite element simulation methods can be used to guide the design process as reported in [20]. Moving from the actuator level to the system level, different actuator configurations are possible with antagonistic configurations enabling simultaneous position and stiffness control (see [21-23]). While certain systems are realized from only soft materials (e.g., [17]), other robotic systems combine soft and rigid materials to form hybrid systems [24].

The modeling of soft robots to derive model-based control strategies is challenging due to the viscoelastic material properties, as reported in [5]. An approach based on a linear parameter-varying model dependent on the input pressure for a continuum robot is presented in [25]. The authors of [26] leverage a reduced order finite element model and an equivalent rigid robot model to describe the dynamics of a continuum robot is 
presented in [27]. Alternatively, the piecewise constant curvature assumption can be used for continuum robots, as documented in [28].

An approach based on sliding mode and fuzzy control is applied in [29] for a rotary actuator with elastic chambers. Model predictive control based on learned nonlinear models as applied in [30] can also be combined with ideas from adaptive control to compensate for model deviations, as presented in [31]. The authors of [32] use reinforcement learning to control a system that is jointly actuated with tendons and pneumatic actuators. Traditionally, the speed at which soft robots move has been limited. However, the authors of [33] demonstrate that fast actuation is possible. Learning techniques such as iterative learning control also show promise to achieve accurate tracking for higher actuation speeds as documented in [19]. The authors of [34] argue that an iterative learning control approach resulting in a feed forward signal allows preservation of the desired compliance of soft robots. On the other hand, applying high gain feedback control to achieve accurate tracking performance would lead to a stiffening of the robot. A combination of low-gain feedback control with a learned feed forward control action is used in [35] to deal with unexpected interactions with the environment and an approach to generalize learned feed forward signals across different execution velocities is presented in [36].

In terms of pick and place applications, the authors of [37] present a soft planar manipulator that is used to manipulate objects in the plane, where [38] demonstrates the grasping of objects with a soft spatial manipulator in a three dimensional space. A collaborative pick and place application has been realized with Festo's Bionic Handling Assistant [16], where an object is either handed over to a human or a second manipulator. A fabric-based manipulator for supporting humans in daily living tasks is presented in [39]. The authors also discuss the challenge of lifting a payload with the horizontally extended manipulator under the effect of gravity. A pick and place application with an inflatable robot arm is presented in [40] in the context of space exploration. Visual servoing is used in an outer control loop to pick objects of different sizes.

The compliance of soft robots makes them particularly suited to human-robot interaction, as reported in [41]. Examples of such systems are wearable soft robots supporting humans in a variety of tasks (see e.g., [42-44]) or devices for rehabilitation [45]. A soft robotic system particularly designed for safe interactions with humans is documented in [46] and the estimation of external contact events for a soft continuum robot is proposed in [47]. A combination of model-based disturbance observation and data-driven techniques is employed to estimate the external forces acting on the robot.

\subsection{Contribution}

In this work, we present a safety analysis of a soft manipulator during object manipulation that motivates the design of the inflatable, and hence lightweight, soft robotic arm. The design of the robot manipulator, including the mechanical, pneumatic, and electronic aspects, is presented in detail and represents a significant improvement compared to the predecessor system discussed in [23]. The current system has been employed for an application in [48], with the design of the system not presented in detail. Leveraging state-of-the-art fabrication techniques, such as high-frequency welding, improves the robustness and reliability of the system. Introducing an axial degree of freedom and a suction cup at the tip of the robot enables the grasping of various objects. The usage of proportional valves with sufficient flow capacity ensures that the actuator pressures can be controlled sufficiently quickly. This ensures a timescale separation from the slower arm dynamics that forms the basis for the applied cascaded control architecture that is a common control strategy for pneumatically actuated soft robots. We present an investigation that reveals that insufficient valve flow capacity prevents a timescale separation and results in oscillatory closed loop behavior. Since soft robotic systems already tend to be under-damped as a consequence of the soft materials employed, a degradation of the overall control performance can result. 
The modeling and control approach is based on the control allocation strategy presented in [48]. An important property of the control allocation strategy is discussed. It ensures equal usage of all actuator pressures and consequently leverage of the full valve flow capacity. While the controller in [23] is based on a numerical pressure to angle relation, a parametric model originating from [48] is used in this work. The systematic modeling approach addresses the effects of changing joint stiffness and the presence of a payload mass on the dynamics. Both influences are relevant due to the lightweight nature of the robotic arm. Simultaneous position and stiffness control is demonstrated with and without a payload mass being attached. The repeatability and overall control performance of the system is clearly improved in comparison to [23], as a consequence of the improved mechanical, pneumatic and electronic design, as well as the systematic modeling and control approach deployed.

While learning control was indispensable in [48] for reliably picking an object, the development of a closed form feed forward strategy allows us to pick an object without using iterative learning control. Two applications are realized where the robot arm picks an object from a known position and places the object at a fixed target location or hands it over to a human. In the second case, an intrinsic property of the robotic arm is exploited: The compliance of the robot arm allows a forced movement by the human that can be detected autonomously by a deviation from the nominal setpoint angles. Based on this criteria, the robot arm releases the object to hand it over to the human.

\subsection{Outline}

The outline of the paper is as follows: The safety analysis motivating the design of the system is presented in Section 2. The design of the robot arm is discussed in Section 3, while Section 4 summarizes the control allocation strategy and the modeling of the system. Section 5 presents the cascaded control strategy deployed and an analysis of the underlying timescale separation. The realizations of different object manipulation applications are presented in Section 6 and a final conclusion is drawn in Section 7.

\section{Safety Considerations}

As a consequence of their intrinsic properties, soft robots have the potential to reduce the danger of an injury caused to a human in the case of an unintended interaction [7]. The compliance of the soft materials employed, as well as the potentially reduced weight of the overall system, are key factors. An investigation of the safety properties of a soft robotic arm intended for object manipulation is presented and used to derive design requirements.

The most closely related systems to soft robots for which safety requirements exist are collaborative robots. The ISO norm 15,066 [49] defines four collaboration methods for the safe deployment of collaborative robots: Either the robot stops when a human enters the moving area of the robot or the robot is moved manually by a human if the moving speed is reduced. Otherwise, the distance between the robot and the human is determined and the speed of the robot is reduced based on this distance criteria. Finally, an interaction between human and robot is possible if certain thresholds for the applied torque, force, power, and speed are not exceeded. The last requirement is particularly relevant as it allows for an interaction with humans under certain circumstances. For soft robots on the other hand, no guidelines currently exist for safe deployment. While the use of soft materials generally improves the safety aspect of soft robots according to [7], the authors also identify three key factors representing a possible source of danger: These are material failure; the so called whiplash effect; and the energy stored in the system. In the case of our system, the first point (material failure) is addressed by the use of advanced fabrication techniques to improve the robustness and reliability of the different components, as discussed in Section 3.2. The whiplash effect describes an acceleration of the tip section of a continuum soft robot when colliding with an obstacle. The radius of the moving part of the robot is reduced and the conservation of angular momentum leads to an acceleration of the tip. A detailed definition in the context of soft robotics is provided in [7]. However, the whiplash 
effect does not apply to our system as the links cannot deflect sufficiently to facilitate this kind of behavior. Finally, the energy stored in the system requires a detailed analysis, where the elastic energy stored in the deformed soft materials and the kinetic energy of the system are primarily relevant. As the elasticity of the materials used for the links and actuators is limited (see Section 3.2), we focus the discussion on the kinetic energy of the system.

Thereby, the interaction forces between the robot manipulator and a human during an unintended collision are analyzed. The scenario, as illustrated in Figure 2 (left plot), forms the basis of the analysis.
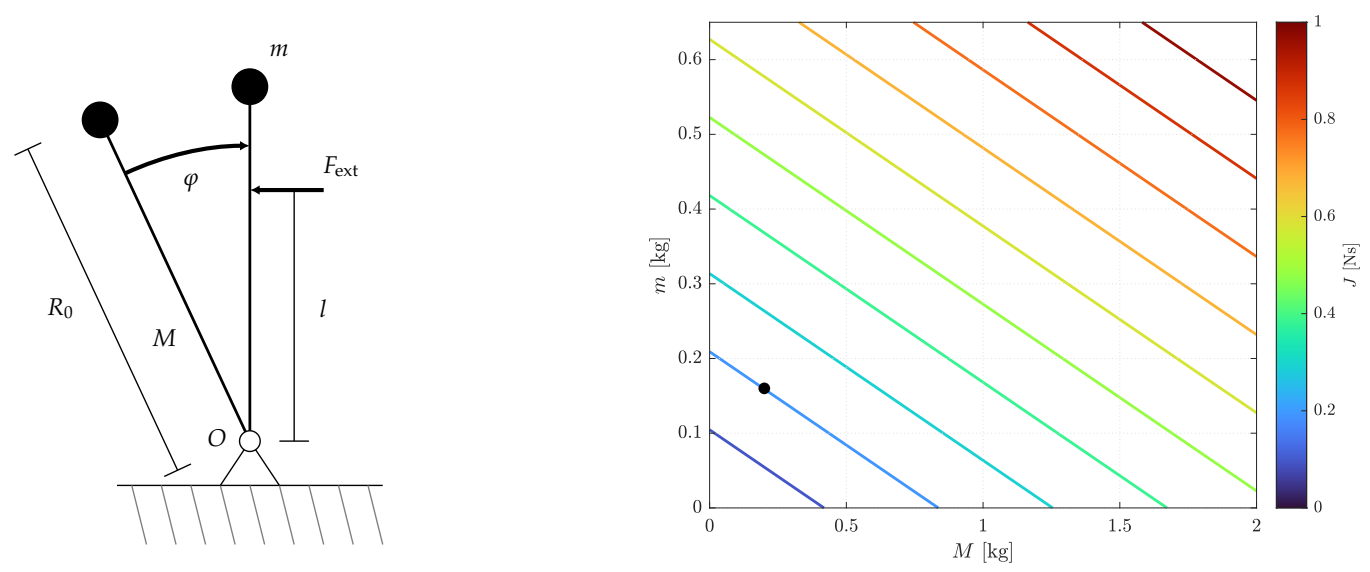

Figure 2. (Left) The scenario considered for investigating the safety aspect is a one degree of freedom manipulator of mass $M$, with a payload of mass $m$ attached to its tip. The mass of the link is assumed to be concentrated as a point mass at the middle of the link. The radius of the link (measured from pivot point $O$ to the tip) is $R_{0}$. The robot collides with a human at a distance $l$ from the pivot point, causing a resulting external force $F_{\text {ext }}$ acting on the movable link and similarly acting on the human. The influence of gravity is not considered in this example. (Right) The momentum $J$ (time integral over external force) as a function of the robot arm mass, $M$, and the payload mass, $m$. The black dot indicates the mass of the robot arm presented in this work $(M=0.2 \mathrm{~kg})$ and a payload mass of $m=0.16 \mathrm{~kg}$ as used in the applications presented in the last part of this work. The distance from pivot point to collision point, $l$, is assumed to be two-thirds of the link radius. The initial angular velocity of the robot arm is $1.8 \mathrm{rad} / \mathrm{s}$ corresponding to the highest angular velocity considered in this article. The momentum grows with the robot link mass and the payload mass. If we want to keep the momentum constant (moving on a colored line) and the mass of the robot arm is increased by a factor of two, the payload mass would need to be reduced by one third.

A one degree of freedom robotic manipulator with a payload mass attached to its tip collides with a human, causing an external force, $F_{\text {ext }}$, acting on the robot and similarly on the human. We assume that the robot arm moves with an initial angular velocity $\dot{\varphi}^{-}$and comes to a complete standstill after the collision with the human $\left(\dot{\varphi}^{+}=0\right)$. Furthermore, the actuation torque driving the robot arm is assumed to be decoupled from the movable link during the interaction due to a sufficiently compliant joint. Applying the laws of impact (see [50]) leads to the following equivalence,

$$
\begin{aligned}
\bar{\Theta}_{0}\left(\dot{\varphi}^{+}-\dot{\varphi}^{-}\right) & =-l \cdot J \quad \text { with } \quad J:=\int_{t^{-}}^{t^{+}} F_{\text {ext }} \cdot d t \\
\Rightarrow J & =\frac{\dot{\varphi}^{-} \bar{\Theta}_{0}}{l} \Leftrightarrow \quad \Leftrightarrow \quad=\frac{\dot{\varphi}^{-} R_{0}^{2}(m+M / 4)}{l},
\end{aligned}
$$

where $t^{-}$and $t^{+}$denote the instants of time before and after the impulse and $\bar{\Theta}_{0}$ is the combined moment of inertia of the robot link and the payload mass wrt. the pivot point $O$. The resulting momentum $J$ for different robot link and payload masses is shown in Figure 2 (right plot). As the length of the robot link and the payload mass are defined 
by the application and the interference point with the human is unknown, we conclude that the only variables to limit the momentum are the initial angular velocity, $\dot{\varphi}^{-}$, or the mass of the robot link, M. Moving at low speeds (as similarly defined in [49]) can easily be implemented, but might also impose restrictive constraints for the application at hand. Hence, the reduction of the robot link mass is identified as the prime design requirement and investigated in detail in Section 3. Note that more complicated interaction scenarios exist, as outlined in [51], are not investigated here. Furthermore, the effect of a compliant link that distributes the interaction force over a larger surface and consequently leads to a reduction of the peak pressure is neglected.

\section{Design}

\subsection{Design Considerations}

The system presented in this work was used in [48] for the realization of an application and originates from [23]. The same actuator configuration is employed, where three actuators allow us to control two rotational degrees of freedom and to simultaneously adjust the joint stiffness. The motivation for the system presented in [23] was to rely on simple fabrication techniques that are commonly available, which means compromising on the robustness of the system. Alternatively, the motivation for the system presented in this work is to design and realize a reliable, robust, and capable system that allows us to realize a pick and place application while addressing the design requirements outlined in Section 2. Therefore, different materials and manufacturing methods are applied that lead to the following improvements compared to the system presented in [23]: The burst pressure of the new bellow-type actuators is increased from 1.5 bar to 6 bar and the angular actuation range from $45^{\circ}$ to $75^{\circ}$. The usage of a soft joint enables an additional translational degree of freedom in the longitudinal direction of the movable link. The tubing is routed internally, and an end effector (suction cup) is integrated to manipulate objects of up to $0.6 \mathrm{~kg}$. The modularity of the overall system is increased and allows us for example to use different suction cups depending on the object being manipulated, or to switch to different actuators with an integrated sensing capability (see [52]). Using proportional valves with sufficient flow capacity improves control authority and ensures a timescale separation between the pressure and arm dynamics (a detailed discussion is presented in Section 5.2). The use of embedded hardware to execute the low level controller and data acquisition improves signal to noise ratio, reduces communication delays in the pressure control loops, and ensures real-time execution of the control approach.

While the use of soft materials can improve dexterity and safety, it can also introduce unwanted effects, such as uncontrollable degrees of freedom, or non-repeatable behavior complicating the control task or even limiting the general capability of the system. Therefore, we consider a systematic design and manufacturing process essential to fully exploit the potential of soft materials, while avoiding potential limitations. The mechanical, pneumatic, and electronic subsystems of the robotic arm are discussed in detail in the following sections.

\subsection{Mechanical System}

In this section, the mechanical part of the inflatable robotic arm is discussed. In order to meet the safety considerations discussed in Section 2, the robotic arm needs to be lightweight. This is addressed by using two inflatable links that are referred to as the static link and the movable link. They are connected by a soft joint that is flexible in both rotational degrees of freedom, and also slightly in the longitudinal direction. Three actuators are arranged symmetrically around the joint and the static link is fixed to a wall by means of a base plate. A suction cup at the tip of the movable link forms the end effector. A rendering of the inflatable robotic arm is shown in Figure 3.

The links consist of a double shell design, where the inner airtight bladder is formed from a polyurethane film (4201 AU $250 \mu \mathrm{m}$ from Platilon). High frequency welding is used to join two layers of the material by clamping them between two electrodes and applying 
a high frequency alternating electromagnetic field. The heating of the material over the full seam thickness eventually causes the two layers to bond together (for a detailed discussion, see [53]). The outer shell is made from ballistic nylon (polyurethane-coated 500 den Cordura fabric from extremtextil), providing high tear resistance. The Sheet Metal tool from SolidWorks is used to project the three-dimensional surfaces of the outer shell to the two-dimensional fabric plane. The pieces are prepared with a laser cutter and processed by sewing. The outer shell transfers the mechanical load acting on the links, where the bending stiffness is significantly increased by the inflatable inner bladder that increases the thickness of the links and consequently the area moment of inertia. An illustration of the materials and components discussed in this section is provided in Figure 4. So-called support cones (3D printed from Formlabs Flexible Resin FLFLGR02) are glued to the conic ends of the links. They connect the links with the soft joint and are used to fix the actuators. An additional support cone is placed at the upper end of the movable link and houses a threaded socket where various suction cups can be mounted. The movable link has a total mass of only $0.2 \mathrm{~kg}$ as a consequence of the inflatable design and the lightweight materials employed.

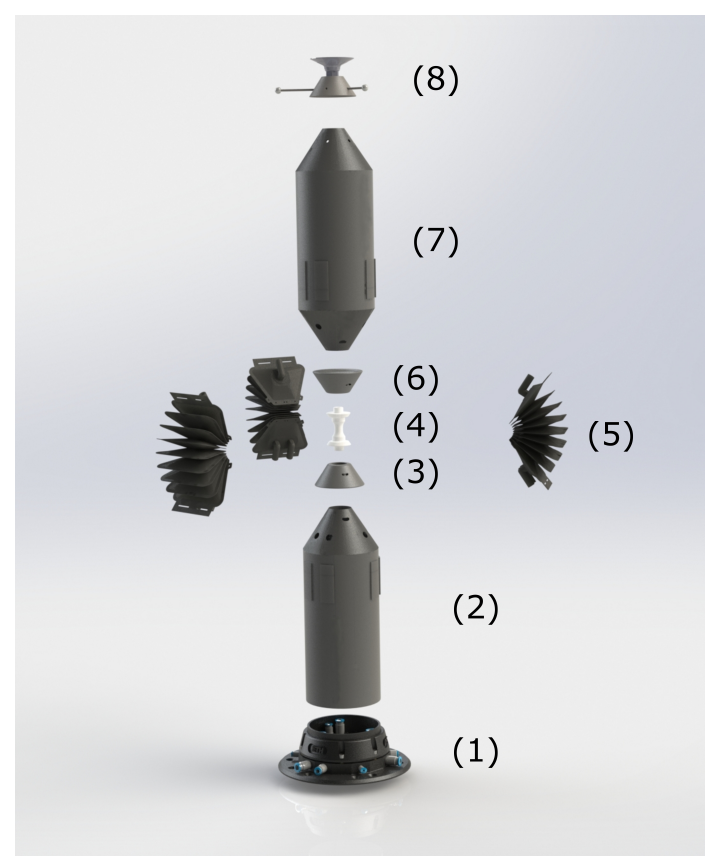

Figure 3. Explosion view of the inflatable robotic arm: The base plate (1) mounts the outer shell of the static link (2) and features tubing connectors that allow to route the tubing internally. The conic support (3) is glued to the interior of the static link and supports the soft joint (4). The three bellow actuators (5) are arranged symmetrically around the soft joint. Tubing is connected through elbow connectors that fit into circular openings in the static and movable links. Additionally, the actuators are fixed by strings attached to the conic supports and Velcro straps on the outer shells of the links. A second conic support (6) connects to the soft joint and is glued to the movable link (7). A third conic support (8) is mounted on the top end of the movable link and houses the suction cup and the markers for the motion capture system. The tubing, the inner bladders, the strings for mounting the actuators, and the screws are not shown for better visibility.

The soft joint is designed to enable a high angular range of the movable link and provide some flexibility in the longitudinal direction to enable the additional translational degree of freedom. Soft silicone elastomer is used (M 4641 from Wacker Elastosil) that features low bending stiffness and intermediate elongation stiffness. The soft joint fits tightly into a recess in the support cones. Tubing to pressurize the movable link and provide a vacuum for the suction cup is routed through a channel in the middle of the soft joint. The diameter of the joint reduces in the middle in order to reduce the bending stiffness and 
the occurring bending stress. The outer diameter is constrained by the minimum diameter of the internal channel, which is $6.5 \mathrm{~mm}$ for routing the tubing.

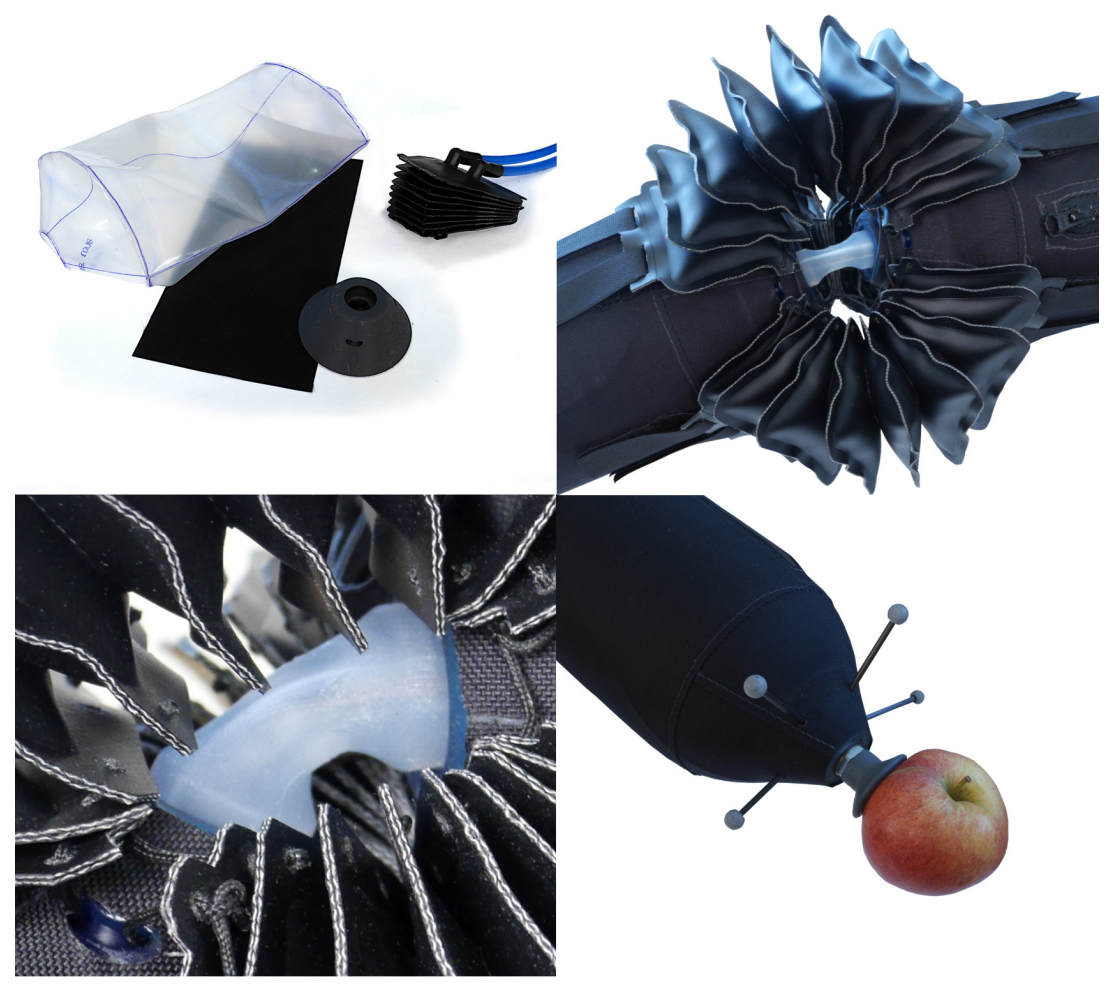

Figure 4. (Top left) The inner bladder of the movable link, the fabric for the outer shell, a support cone with the recess in the middle into which the soft joint fits, and an actuator with the tubing connected. (Top right) The actuators arranged around the soft joint (white cylindrical part) and connected to both links. (Bottom left) The deformed soft joint when the movable link is deflected. The actuators are attached to the support cones with strings. (Bottom right) The tip of the movable link allows us to attach different suction cups to grasp different objects, such as an apple. Markers for the motion capture system are attached for sensory feedback. The markers and the suction cup are mounted to the support cone that is glued to the interior side of the movable link tip.

The actuators aim to convert pressurized air into an angular deflection that is used to actuate the movable link. Each actuator consists of multiple cushions that are connected by an inner seam. Placing the seam off-center allows the actuator to deform in an angular direction. The form of the inflated actuator in free space, i.e., when not mounted to the system, should match the constrained form when mounted to the system as closely as possible. Any difference between the constrained and unconstrained deformation leads to stress imposed on the mounting structure of the actuator. For the deformation of the actuator, the off-center distance of the inner opening, $\mu$ and the height of the actuator, $\rho$ are important (see Figure 5). These parameters are determined through an iterative design process, where an in-house manufacturing technique based on a thermal heat press is applied. For a detailed discussion of the in-house manufacturing technique, the reader is referred to [23]. The in-house fabrication process is inexpensive and allows rapid prototyping, while the final design iteration is realized with high frequency welding to provide robust and reliable actuators. The material for the final design iteration is nylon fabric coated with thermoplastic polyurethane (Riverseal 842 from Rivertex). The material and manufacturing process is also used in [19], with different properties improved, such as the expansion behavior and the tear resistance. For the actuators, the welding machine operates at a frequency of $27 \mathrm{MHz}$ and outputs a power of $1700 \mathrm{~W}$. The applied normal force is $1600 \mathrm{~N}$ for the outer seam with a welding time of $5 \mathrm{~s}$ followed by a cool down time of $5 \mathrm{~s}$ where the electrode is left closed. The electrode for welding the outer seam is 
slightly higher at the inner circumference $(0.1 \mathrm{~mm})$ to form a weld bead for improving tear resistance. Two right angle tubing flanges $\left(90^{\circ}\right.$ small tubing flange from Halkey Roberts) are connected to the bottom side of the actuator. One flange is used to connect the valve and a second tube is used to connect the pressure sensor. An additional tubing flange is welded to the top side of the actuator for precise positioning with respect to the movable link. Circular openings in the outer shell of both links allow the routing of tubing flanges through the shells. The tubing for the air supply and the pressure sensor is routed between the inner bladder and the outer shell of the static link to the base plate. The actuator is fixed with strings to the support cones and with Velcro straps to both links.
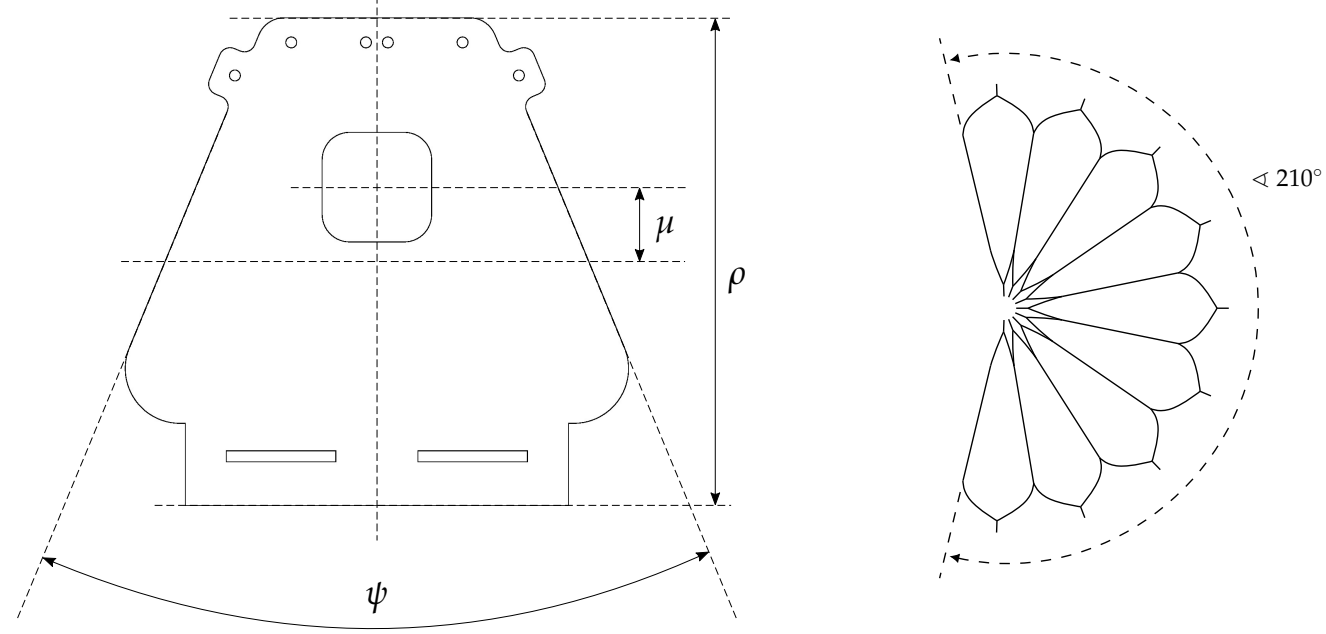

Figure 5. The left figure shows the top view of a single actuator layer. The deformation behavior mainly depends on the actuator height $\rho$ and the off-center distance $\mu$. Choosing the angle $\psi>0$ increases the footprint of the actuator and therefore the lateral stability during inflation such that the actuator does not bend sideways. The following parameter values are used for the final design: $\rho=89 \mathrm{~mm}, \mu=13.6 \mathrm{~mm}$, and $\psi=45^{\circ}$. The figure on the right shows the side view of an inflated actuator. The nine cushions result in a total angle of approximately $210^{\circ}$. The outer arc length of the actuator when fully inflated is approximately $340 \mathrm{~mm}$, compared to a thickness of $18 \mathrm{~mm}$ when the actuator is fully collapsed.

The base plate is made from rigid materials (3D printed from PA12) as it is neither moving nor sticking out from the table on which it is mounted. The outer shell of the static link is sandwiched between the inner part of the base plate and an outer ring that can be fastened to the base. Weld-in sleeves are placed on the top and on the side of the base plate to connect all required peripherals.

\subsection{Pneumatic System}

In this section, the pneumatic subsystem of the robotic arm is discussed. The requirements for the pneumatic system include control of the air pressure in the three actuators, to inflate both links to a static air pressure and to provide sensory feedback of the actuator and source pressures. Furthermore, since pressurized air is already in use for actuating the system, a vacuum suction cup is used for grasping objects. Different safety features are included to ensure that no maximum pressure level for any of the components is exceeded. The detailed pneumatic diagram is shown in Figure 6.

Different types of valves are available to control the air pressures in the bellow actuators. Binary switching valves were used to operate the system presented in [23]. These are directional valves that are either fully open or fully closed. Applying a pulse width modulation strategy allows us to continuously adjust the air flow. Proportional directional valves on the other hand can continuously adjust the spool position and therefore the air flow. A second important property of the valve is its air flow capacity. It determines the maximum possible air flow through the valve and consequently the bandwidth of the 
pressure dynamics in the actuator. For the system presented in this work, proportional directional control valves (MPYE-5-1/4-010-B from Festo) with a standard nominal flow rate of $1400 \mathrm{~L} / \mathrm{min}$ at reference conditions (defined by DIN 1343 as entry pressure to the valve of 6 bar and exit pressure of 5 bar) are used. The flow capacity is more than four times larger compared to the system presented in [23], and therefore enables fast control of the actuator pressures. Implications of the valve flow capacity on the control performance will be investigated in detail in Section 5.2. In addition to the valve flow capacity, the available source pressure and the actuator volume also affect the bandwidth of the pressure dynamics. The inflation of the actuators can be accelerated by using a higher source pressure, while the deflation is limited by the ambient air pressure. The actuator volume is given by the design presented in Section 3.2, where a small actuator volume is a secondary design objective.

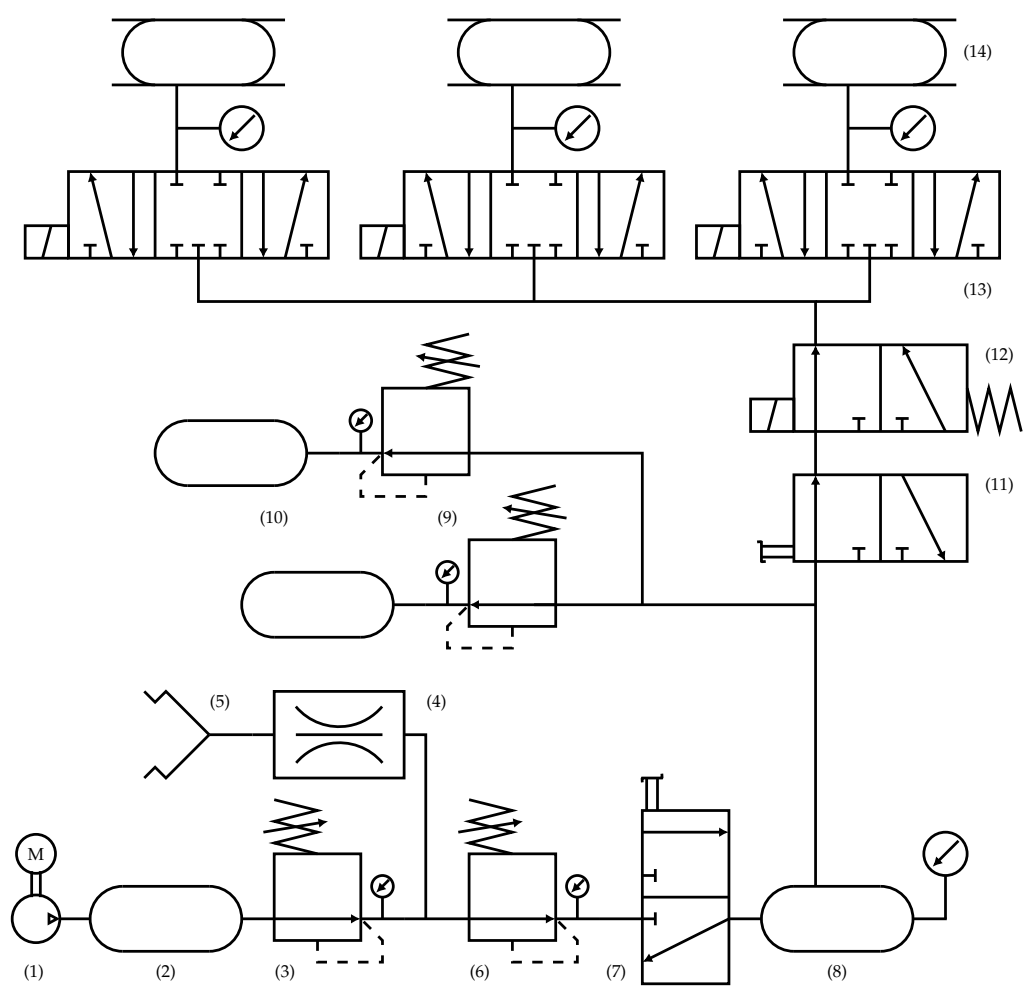

Figure 6. The pneumatic diagram of the soft robotic arm: A compressor (1) provides pressurized air at 9 bar that is fed to an air receiver (2). The pressure level is reduced to 7.5 bar by means of a manual pressure regulator (3) to the level used by the vacuum generating unit (4) to operate the suction cup (5). A second manual pressure regulator (6) decreases the pressure level to $3.5 \mathrm{bar}$, which is the pressure level used to operate the proportional valves controlling the actuator pressures. A manual shut off valve (7) allows us to either supply air to the additional receiver (8) or to exhaust the air of the subsequent system (as shown in the current valve position). The receiver (8) allows us to mitigate air flow delays induced by the preceding components. The pressure in receiver (8) is measured by means of a pressure sensor and referred to as the source pressure. Two additional manual pressure regulators (9) allow us to adjust the air pressure in the static and movable links (10) (approximately 0.25 bar). An additional shut off valve (11) can be used to cut off the air supply from the actuators, while maintaining pressurization of the links. A safety valve (12) ensures that the actuators are exhausted in the case of an emergency. The valve is normally closed, meaning that it has to be actively opened by its solenoid (configuration shown) to supply air to the actuators. Three proportional directional valves (13) are used to control the air pressure in the three actuators (14), where each pressure is measured for feedback control. Note that only three of the five ports of each proportional valve are in use. 
For operation of the suction cup, a vacuum generation device is required. A vacuum generator based on the Venturi effect is employed (OVEM-14-L-B-QO-CE-N from Festo). The unit includes two independent solenoid valves. The primary valve operates the Venturi nozzle to generate a vacuum and the secondary valve operates an ejector pulse to quickly release the vacuum in the suction cup. The two control inputs are typically operated in the following way: for the duration of the object being grasped, the primary valve is opened to generate a vacuum. As soon as the object should be released, the primary valve is closed and the secondary valve is opened for a short time duration to rapidly release the vacuum in the suction cup. For safety reasons, an additional binary directional valve with high flow capacity is placed before the proportional valves operating the actuators. It is normally closed and needs to be actively opened by a solenoid. If the power is shut off when using the emergency button, the valve automatically closes and exhausts the air supply to the proportional valves. Simply switching off the power to the proportional valves only brings them to their neutral position. However, due to leakage of the valves, the actuator pressure could still increase, presenting a possible risk.

\subsection{Electronic System}

An embedded hardware and an additional laptop computer are used to operate the inflatable robotic arm. An embedded system (STM32 Nucleo-144 development board with STM32F413ZH MCU from STMicroelectronics) with a custom interface board meets the sensing, communication and low level computation requirements. The pressure sensors are interfaced over analog-to-digital converters sampled at $200 \mathrm{kHz}$. This allows us to average the raw analog-to-digital readings and provide pressure measurements at $1000 \mathrm{~Hz}$ with a high signal to noise ratio. The proportional valves are interfaced over analog outputs, realized by low-pass filtering pulse width modulated signals. The vacuum unit is interfaced over two digital outputs switching the two solenoid valves. The low level controllers for operating the pressure controllers are executed on the embedded hardware at $1000 \mathrm{~Hz}$. Communication between the embedded hardware and the laptop computer is realized by means of serial communication.

In comparison to the setup employed in [23], using embedded hardware has the advantage that communication delays in the pressure control loop can be reduced. The sensing of the actuator pressures, filtering, execution of the low level controller, and outputting the commands to the valves is all deployed on a single piece of hardware. The schematics (see Supplementary Materials S2) of the custom interface board are provided in the Supplementary Materials.

The laptop computer is connected to an infrared motion capture system (from Vicon) running at $200 \mathrm{~Hz}$ and providing positional data with sub-millimeter accuracy. High level tasks, including a graphical user interface, are executed on the computer. More details about the control architecture are provided in Section 5.2.

\section{Modeling}

In this section, the kinematic and dynamic description of the soft robotic arm is presented. First, the end effector parametrization is introduced. Then, the control allocation strategy is outlined, where different possible strategies are compared. The control allocation strategy forms the basis for the dynamical modeling approach and permits us to consider the two angular degrees of freedom as decoupled. Subsequently, limitations in the modeling of the arm dynamics related to viscoelastic material properties are highlighted and the section is concluded with an analysis of how an elongation in the longitudinal direction affects the rotational degrees of freedom.

\subsection{End Effector Parametrization}

The robotic arm has three actuators and the pressure in each actuator can be controlled independently, forming the three control inputs. The end effector can move on a spherical cap described by two degrees of freedom. As presented in [23], the additional control input 
allows us to adjust the joint stiffness as well as the control of the two rotational degrees of freedom (see Figure 7). Differently, to the system presented in [23], a soft joint is used in this work. As a consequence, increasing the joint stiffness by inflating all three actuators simultaneously results in a longitudinal elongation of the soft joint, as shown in [48]. This effect can be used for grasping an object. However, it is important to mention that the joint stiffness and the longitudinal elongation can not be controlled independently.
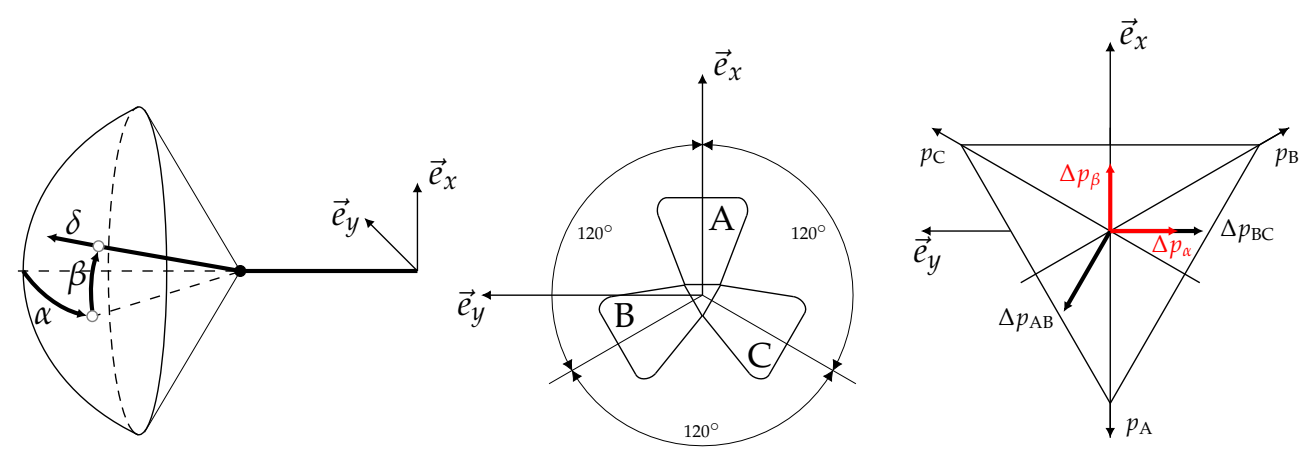

Figure 7. (Left) The spherical robot arm when mounted horizontally to a wall. The soft joint is indicated by the black circle and the gravitational vector points in the negative $\vec{e}_{x}$ direction. The end effector point is parameterized by two extrinsic Euler angles $\alpha, \beta$, both describing rotations with respect to the inertial frame, and the variable $\delta$ describing a longitudinal elongation. (Middle) The symmetric actuator configuration with the three actuators $\mathrm{A}, \mathrm{B}$, and $\mathrm{C}$ in the corresponding coordinate system. (Right) The variables used by the control allocation strategy. Note that an increase in the actuator pressure $\mathrm{A}$ acts in the negative $x$-direction.

\subsection{Control Allocation}

For the given actuator configuration, it was shown in [23] that adjusting the lower pressure level allows us to control the joint stiffness, which was experimentally validated for discrete joint stiffness levels. A continuous mapping for the joint stiffness that decouples the rotational degrees of freedom from the joint stiffness was presented in [48] and proven in [54]. We refer to this strategy as the control allocation as it maps the three original actuator pressures $\left(p_{\mathrm{A}}, p_{\mathrm{B}}, p_{\mathrm{C}}\right)$ to three virtual control inputs $\left(\Delta p_{\alpha}, \Delta p_{\beta}, \bar{p}\right)$, where $\Delta p_{\alpha}$ only affects the $\alpha$-direction, $\Delta p_{\beta}$ only affects the $\beta$-direction and $\bar{p}:=\min \left\{p_{\mathrm{A}}, p_{\mathrm{B}}, p_{\mathrm{C}}\right\}$ denotes the lower actuator pressure level affecting joint stiffness (or longitudinal elongation). In the following, the key equations of the control allocation strategy are summarized. Then, we compare different choices of virtual control input variables that reveals an important property motivating the above choice. A more detailed derivation (see Supplementary Materials S3) of the control allocation strategy, including a visualization to provide more intuition, can be found in the Supplementary Materials.

From the actuator configuration shown in Figure 7, we can see that the two rotational degrees of freedom, $\alpha$ and $\beta$, are affected by the actuator pressures in the following way,

$$
\begin{aligned}
& \alpha \sim \frac{\sqrt{3}}{2} p_{\mathrm{B}}-\frac{\sqrt{3}}{2} p_{\mathrm{C}}=\frac{\sqrt{3}}{2}\left(p_{\mathrm{B}}-p_{\mathrm{C}}\right) \\
& \beta \sim-p_{\mathrm{A}}+\frac{1}{2} p_{\mathrm{B}}+\frac{1}{2} p_{\mathrm{C}}=-\left(p_{\mathrm{A}}-p_{\mathrm{B}}\right)-\frac{1}{2}\left(p_{\mathrm{B}}-p_{\mathrm{C}}\right) .
\end{aligned}
$$

Motivated by this relation and by introducing the variables $\Delta p_{\mathrm{AB}}=p_{\mathrm{A}}-p_{\mathrm{B}}$ and $\Delta p_{\mathrm{BC}}=p_{\mathrm{B}}-p_{\mathrm{C}}$, the virtual control inputs $\Delta p_{\alpha}$ and $\Delta p_{\beta}$ are expressed in terms of the actuator pressure differences,

$$
\left[\begin{array}{l}
\Delta p_{\alpha} \\
\Delta p_{\beta}
\end{array}\right]=\left[\begin{array}{cc}
0 & \sqrt{3} / 2 \\
-1 & -1 / 2
\end{array}\right]\left[\begin{array}{l}
\Delta p_{\mathrm{AB}} \\
\Delta p_{\mathrm{BC}}
\end{array}\right] \Leftrightarrow\left[\begin{array}{l}
\Delta p_{\mathrm{AB}} \\
\Delta p_{\mathrm{BC}}
\end{array}\right]=\left[\begin{array}{cc}
-1 / \sqrt{3} & -1 \\
2 / \sqrt{3} & 0
\end{array}\right]\left[\begin{array}{l}
\Delta p_{\alpha} \\
\Delta p_{\beta}
\end{array}\right]
$$


In combination with the definition of $\bar{p}$, this defines the mapping from the actuator pressures to the virtual control inputs,

$$
\left(p_{\mathrm{A}}, p_{\mathrm{B}}, p_{\mathrm{C}}\right) \rightarrow\left(\Delta p_{\alpha}, \Delta p_{\beta}, \bar{p}\right) .
$$

The inverse mapping from virtual control inputs to the three actuator pressures,

$$
\left(\Delta p_{\alpha}, \Delta p_{\beta}, \bar{p}\right) \rightarrow\left(p_{\mathrm{A}}, p_{\mathrm{B}}, p_{\mathrm{C}}\right)
$$

is given by,

$$
\begin{aligned}
& p_{\mathrm{A}}=\max \left\{\bar{p}, \bar{p}+\Delta p_{\mathrm{AB}}, \bar{p}+\Delta p_{\mathrm{AB}}+\Delta p_{\mathrm{BC}}\right\} \\
& p_{\mathrm{B}}=\max \left\{\bar{p}, \bar{p}+\Delta p_{\mathrm{BC}}, \bar{p}-\Delta p_{\mathrm{AB}}\right\} \\
& p_{\mathrm{C}}=\max \left\{\bar{p}, \bar{p}-\Delta p_{\mathrm{BC}}, \bar{p}-\Delta p_{\mathrm{AB}}-\Delta p_{\mathrm{BC}}\right\},
\end{aligned}
$$

with $\Delta p_{\mathrm{AB}}$ and $\Delta p_{\mathrm{BC}}$ being defined in (2) forming functional composition,

$$
\left(p_{\mathrm{A}}, p_{\mathrm{B}}, p_{\mathrm{C}}\right)=\xi\left(\Delta p_{\alpha}, \Delta p_{\beta}, \bar{p}\right),
$$

which is a bijective mapping that can similarly be applied to the virtual control inputs, the absolute pressures (inverse mapping), and the set points of both. In the following, the decoupling between the two rotational degrees of freedom and the joint stiffness is experimentally validated. Therefore, sinusoidal setpoints for $\Delta p_{\alpha}$ and $\Delta p_{\beta}$ are commanded that are tracked by independent pressure controllers (as will be discussed in Section 5.2). The same experiment is repeated for different values of $\bar{p}$. The resulting actuator pressures, the virtual control inputs, and the angular responses are visualized in Figure 8.

Next, we investigate three different virtual control input parametrizations and the implications of this for the actuator pressure usage. Thereby, the virtual control inputs, $\left(\Delta p_{\alpha}, \Delta p_{\beta}\right)$, are used for all three parametrizations due to their direct coupling to the angular degrees of freedom. The third virtual control input is different for each parametrization and summarized in Table 1. The formulas for computing the actuator pressures for each parametrization are given by the formulas in Table 1, Equations (2) and (5), and the definitions of $\Delta p_{\mathrm{AB}}$ and $\Delta p_{\mathrm{BC}}$.

Table 1. Different virtual control input parametrizations: The virtual control inputs $\Delta p_{\alpha}, \Delta p_{\beta}$ are used for all three parametrizations, but the third virtual control input is different for each choice.

\begin{tabular}{cc}
\hline Parametrization & 3. Virtual Control Input \\
\hline 1 & $p_{\text {avg }}=\frac{1}{3}\left(p_{\mathrm{A}}+p_{\mathrm{B}}+p_{\mathrm{C}}\right)$ \\
2 & $\bar{p}=\min \left\{p_{\mathrm{A}}, p_{\mathrm{B}}, p_{\mathrm{C}}\right\}$ \\
3 & $p_{\mathrm{A}}=$ const. \\
\hline
\end{tabular}

The first parametrization is intuitive to understand with the average actuator pressure as the third virtual control input. The second parametrization originates from [23] and is used in this work. The third parametrization is investigated, as it showcases an important property of the different parametrizations possible.

We consider periodic and symmetric input signals in $\Delta p_{\alpha}$ and $\Delta p_{\beta}$ for all three cases,

$$
\Delta p_{\alpha}(t)=0.2 \cdot \sin (2 \pi \cdot t / T), \quad \Delta p_{\beta}(t)=0.2 \cdot \cos (2 \pi \cdot t / T) \quad \text { with } T=1 \mathrm{~s}, t \in[0,2 \mathrm{~s}] .
$$



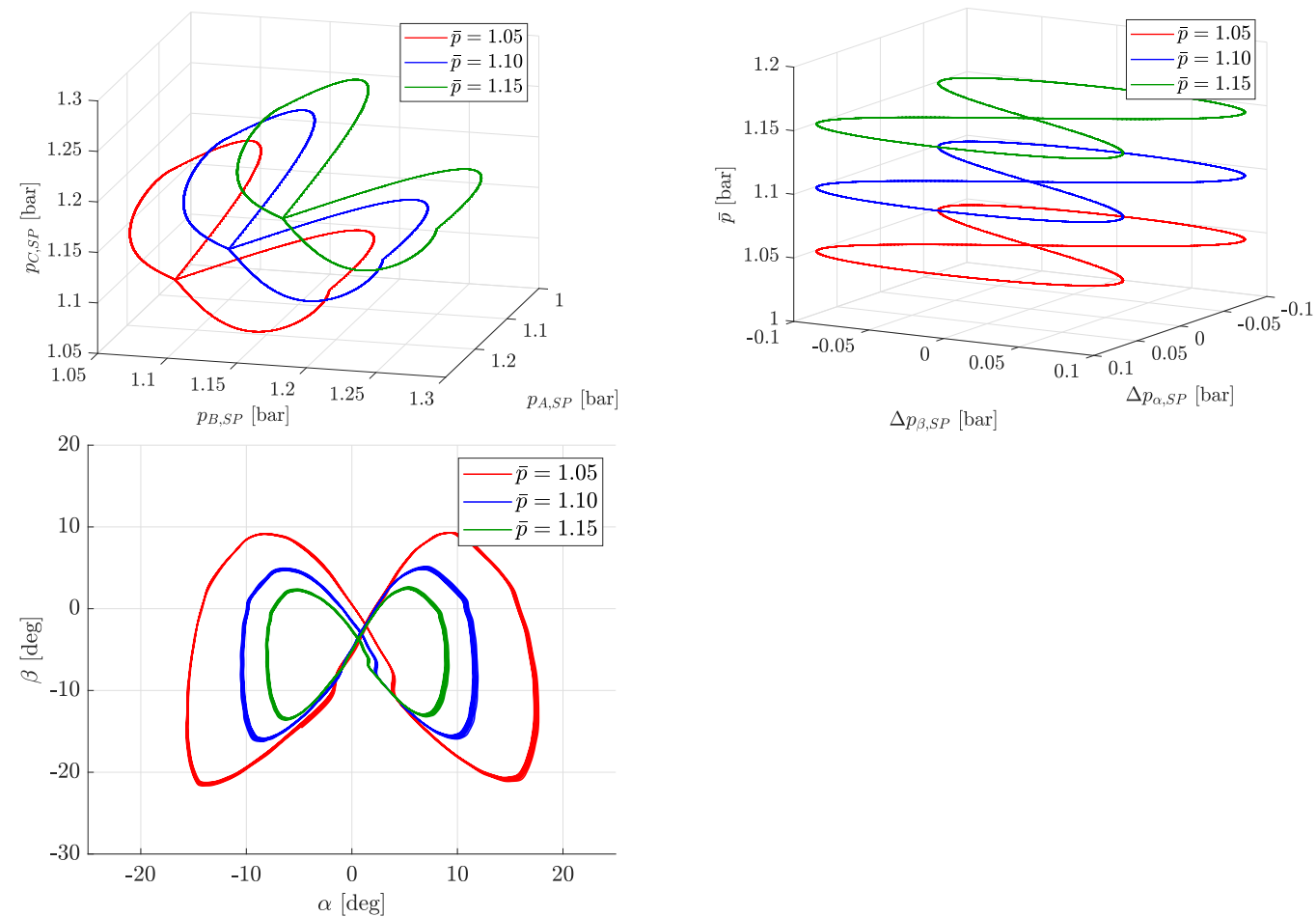

Figure 8. The figure shows a visualization of the control allocation strategy. (Top left) The plot shows the pressure setpoints in the absolute representation, (top right) the virtual control inputs and (bottom) the resulting angular trajectories. Each of the three trajectories has a constant lower pressure level that increases from the red curve ( $\bar{p}=1.05$ bar) to the blue curve $\bar{p}=1.10$ bar and to the green curve $(\bar{p}=1.15$ bar $)$. The setpoint trajectory for the virtual control inputs defines a figure-eight trajectory with a different vertical offset corresponding to different lower pressure levels that result in figure-eight trajectories in the angular space of varying magnitude. Each of the three virtual control input trajectories has the same magnitude, but results in a decreasing angular magnitude for higher values of $\bar{p}$. The reason for this behavior is that the movable link is forced towards a straight orientation wrt. the static link for increasing values of $\bar{p}$ leading to a decrease in angular magnitude. While the set point trajectories have a simple form in the virtual control input representation, the resulting absolute pressure setpoints are rather complex, emphasizing the importance of the control allocation strategy.

A visualization of the virtual control input parametrizations and the resulting actuator pressures $p_{\mathrm{A}}, p_{\mathrm{B}}$ and $p_{\mathrm{C}}$ is provided in Figure 9.

Given periodic input signals for $\Delta p_{\alpha}$ and $\Delta p_{\beta}$, only parametrizations 1 and 2 result in equivalent actuator pressure signals, in the sense that $p_{\mathrm{A}}, p_{\mathrm{B}}$ and $p_{\mathrm{C}}$ are periodic signals of equal magnitude. The reason is that the third virtual control input of parametrizations 1 and 2 is symmetric in all three actuator pressures, which is clearly not the case for parametrization 3 . There are different reasons why equivalent actuator pressure signals are beneficial: First, they guarantee that all valves are equally used, meaning that the available valve flow capacity (of all three valves) is fully exploited. This becomes particularly relevant when considering the implication of the valve capacity on the closed-loop pressure dynamics (see Figure 15 for more details). Secondly, it means that the actuators and valves are used equally, implying that all wear and aging phenomena evolve equally for the three subsystems. Therefore, the control allocation parametrizations 1 and 2 are preferential to parametrization 3 . Note that parametrization 3 would be an interesting allocation strategy for a system where one of the three valves has a significantly smaller flow capacity and could be used to adjust this mainly constant pressure. As parametrization 2 features a virtual control input, $\bar{p}$, with a clear physical interpretation as the joint stiffness (see [23]), it is chosen as the control allocation strategy for this work. 

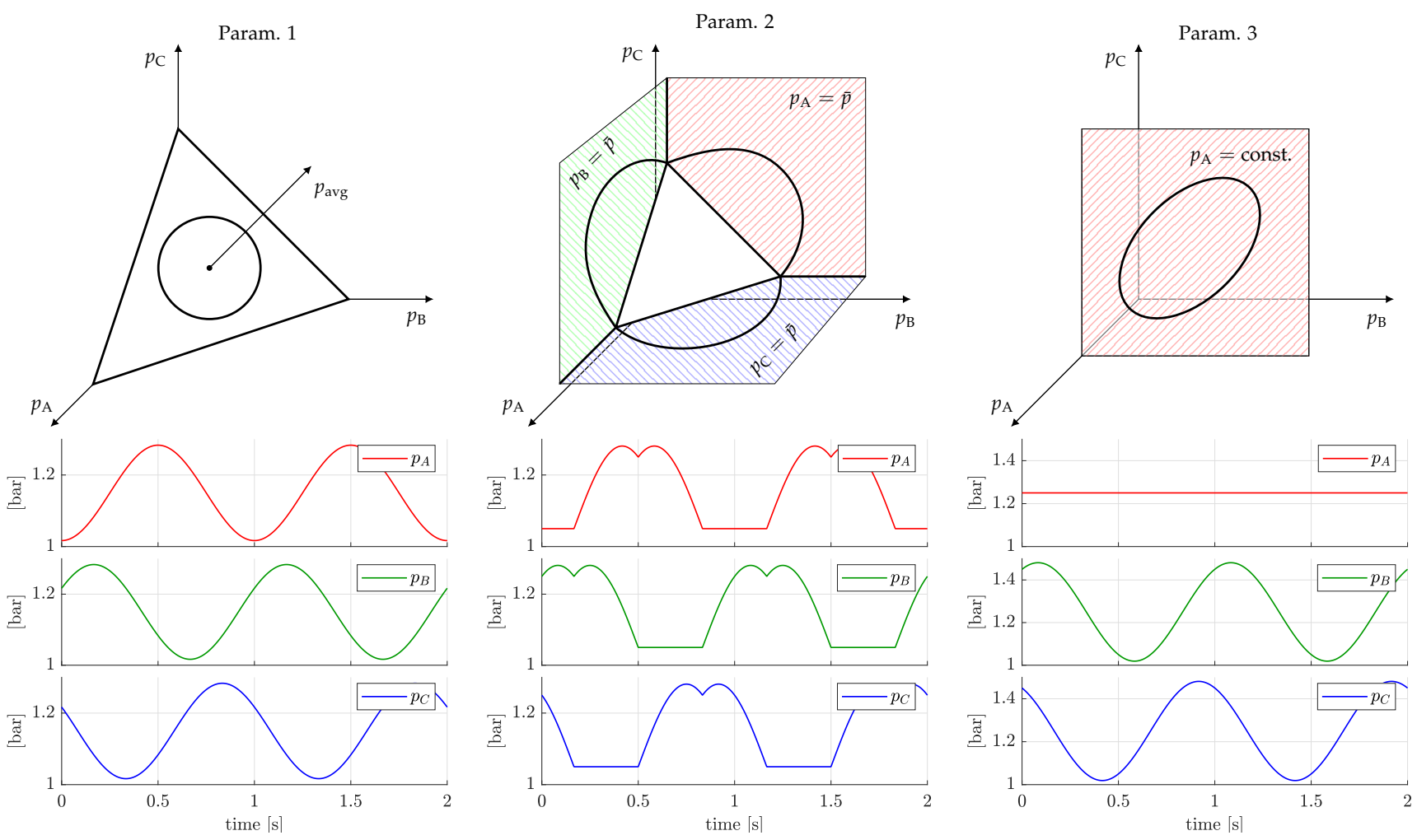

Figure 9. Visualization of the three virtual control input parametrizations: The first column shows parametrization 1 with the average actuator pressure used as the third virtual control input, the second column shows parametrization 2 with the lower actuator pressure level as third virtual control input (as used in this work) and the right column shows parametrization 3, where $p_{\mathrm{A}}$ is directly used as the third virtual control input. The periodic input signal in $\Delta p_{\alpha}$ and $\Delta p_{\beta}$ is indicated for parametrization 1 by the black circle in the pressure space. The virtual control input, $p_{\text {avg }}=1.15 \mathrm{bar}$, is a measure of the distance between the $\Delta p_{\alpha}-\Delta p_{\beta}$-plane containing the circle and the origin. The resulting actuator pressures are shown in the bottom plot and it can clearly be seen that the resulting signals are periodic and of equal magnitude with a shifted phase. For parametrization 2, the resulting curves in the pressure space are given by the black curves (section of an ellipse), lying on the three colored planes that are offset to the origin by $\bar{p}=1.05$ bar. They result from projecting the circle of the input signals, $\Delta p_{\alpha}$ and $\Delta p_{\beta}$, onto the three planes. The three actuator pressures in the bottom plot are also periodic signals with equal magnitude and a shift in phase. For parametrization 3, the curve in the pressure space is obtained by constraining the circle in $\Delta p_{\alpha}$ and $\Delta p_{\beta}$ to the plane $p_{\mathrm{A}}=1.25 \mathrm{bar}$, resulting in an elliptical curve. The corresponding actuator pressures are all periodic signals, but only $p_{\mathrm{B}}$ and $p_{\mathrm{C}}$ have equal magnitude. Hence, this virtual control input parametrization differs qualitatively from parametrizations 1 and 2.

\subsection{Arm Dynamics}

The dynamics are strongly dependent on the lower pressure level as it relates to the joint stiffness and to the attached payload due to the lightweight nature of the system. Consequently, the modeling approach presented in this section needs to address both dependencies to provide a sufficiently accurate basis for the control approach. The applied procedure is twofold: The dependency of the dynamics on the attached payload mass is addressed by relying on a gray box model structure, where the effect of the attached mass is described from first principles. The parameters of the gray box model are identified from system identification experiments that are performed for different lower pressure levels, $\bar{p}$. Low order polynomials as a function of $\bar{p}$ are fitted for each parameter in a second step.

Leveraging the control allocation strategy presented in the previous section allows us to model the arm dynamics as two decoupled systems in the $\alpha$ and $\beta$-direction, respectively. Thereby, $\Delta p_{\alpha, \mathrm{SP}}$ and $\Delta p_{\beta, \mathrm{SP}}$ form the inputs to each system and $\alpha$ and $\beta$ are the outputs. The modeling procedure is discussed for the $\alpha$-direction, but is similarly applied for the 
$\beta$-direction. The corresponding plots can be found in Appendix A. The dynamics are assumed to be given by the (linearized) pendulum equation,

$$
\left(m+\frac{M}{4}\right) R_{0}^{2} \ddot{\alpha}+d_{\alpha} \dot{\alpha}+k_{\alpha} \alpha=\tau_{\alpha} .
$$

The parameter $R_{0}$ denotes the length from the pivot point to the load mass, $m$, when the soft joint is not elongated. The mass of the movable link is given by $M$ and considered as a point mass with distance $R_{0} / 2$ from the pivot point. The stiffness and damping parameters $k_{\alpha}, d_{\alpha}$ are determined through system identification experiments. The equation holds for the robotic arm mounted horizontally to a wall as shown in Figure 7. In case the robotic arm is mounted vertically on a horizontal plane, the equation changes slightly. The dependency between the driving torque, $\tau_{\alpha}$, and $\Delta p_{\alpha, \mathrm{SP}}$ is assumed to be given by a linear first order model,

$$
\tau_{\alpha}(s)=\frac{\eta_{\alpha}}{T_{\alpha} s+1} \Delta p_{\alpha, \mathrm{SP}}(s),
$$

where $s$ denotes the Laplace variable and $\eta_{\alpha}$ and $T_{\alpha}$ the gain and time constant determined from system identification. The first order model accounts for the dynamics between the commanded pressure setpoint and the torque acting on the system. A detailed investigation of the timescale separation of the pressure dynamics and the arm dynamics is presented in Section 5.2. Combining the second order model (8) with the first order actuator dynamics (9) yields the linear, third order model with input $\Delta p_{\alpha, S P}$ and output $\alpha$,

$$
G_{\alpha}(s, m)=\frac{\eta_{\alpha}}{T_{\alpha} s+1} \frac{1}{(m+M / 4) R_{0}^{2} s^{2}+d_{\alpha} s+k_{\alpha}},
$$

and analogously for $\beta$. Note the parametric dependency on the payload mass $m$.

Next, system identification experiments are conducted for different values of $\bar{p}$. Thereby, no payload mass is attached. A series of sinusoidal excitation signals are applied sequentially in $\Delta p_{\alpha, \mathrm{SP}}$ and $\Delta p_{\beta, \mathrm{SP}}$, respectively. The lower pressure level, $\bar{p}$, is set to a fixed value for each identification experiment in the range of $1.0 \mathrm{bar}$ to $1.2 \mathrm{bar}$. For each frequency, the input signal is applied for ten successive periods, where the first four periods are discarded to reduce transient effects. The remaining periods are averaged and the magnitude and phase of the transfer function are estimated using a sinusoidal correlation method (see [55]). A continuous-time transfer function with three poles and no zero, as given by the model structure (10), is fitted to the estimated data. The measured transfer function and the resulting fit for the $\alpha$-direction are shown in Figure 10 for the different values of $\bar{p}$.

The frequency responses show a clear dependency on the lower pressure level $\bar{p}$. In order to address this dependency in the model, the gain, time constant, stiffness, and damping parameters in (10) are assumed to depend on $\bar{p}$, namely

$$
G_{\alpha}(s, \bar{p}, m)=\frac{\eta_{\alpha}(\bar{p})}{T_{\alpha}(\bar{p}) s+1} \frac{1}{(m+M / 4) R_{0}^{2} s^{2}+d_{\alpha}(\bar{p}) s+k_{\alpha}(\bar{p})} .
$$

A comparison of the coefficients for each of the five identification experiments performed for a certain lower pressure level, $\bar{p}$, provides a set of parameter values for $\eta_{\alpha}$, $T_{\alpha}, d_{\alpha}$, and $k_{\alpha}$. Low order polynomials are fitted to the five values for each parameter separately to obtain a parametric description in $\bar{p}$, as shown in Figure 11.

Combining the parametric dependency on the lower pressure level with the dependency on the payload mass from first principles results in a linear parameter varying model in $\bar{p}$ and $m$. Note that the approach applied here differs from the method presented in [25], where the linear parameter varying model depends on the input pressure and not on a lower pressure level. 

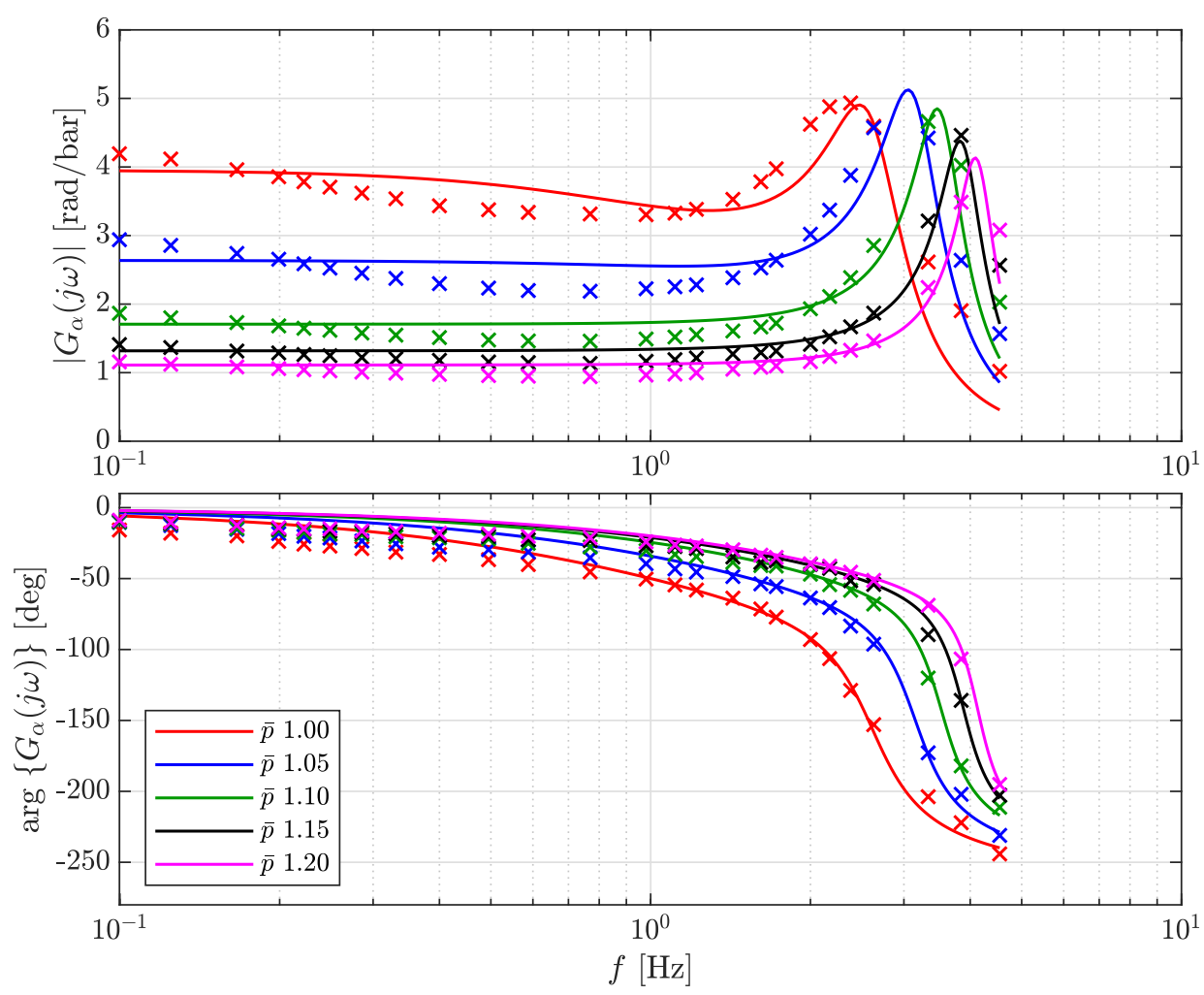

Figure 10. The frequency response in the $\alpha$-direction for different lower pressure levels $\bar{p}$ with the magnitude in the top plot and the phase in the bottom plot. The measured frequency responses resulting from the identification experiments are indicated by the crosses and the corresponding fits by the solid lines. For increasing values of $\bar{p}$, the magnitude of the frequency response decreases and the resonance frequency of the system increases. The error between measured and fitted frequency response could be further reduced for small values of $\bar{p}$ by adding an additional zero to the fitted transfer function. However, we reject the extension for the sake of simplicity and in order to have a constant model structure for all values of $\bar{p}$.

During the identification experiments, no payload mass was attached. In order to validate the model for non-zero payload masses, two additional system identification experiments are conducted in $\alpha$-direction, where the same procedure as before is applied, but a mass of $0.1 \mathrm{~kg}$ or $0.2 \mathrm{~kg}$ is attached. The lower pressure level is set to an average value of 1.1 bar for both experiments. A comparison between the model prediction and the actual measured frequency response is shown in Figure 12, showing that the model predicts the change in the dynamics relatively accurately. 

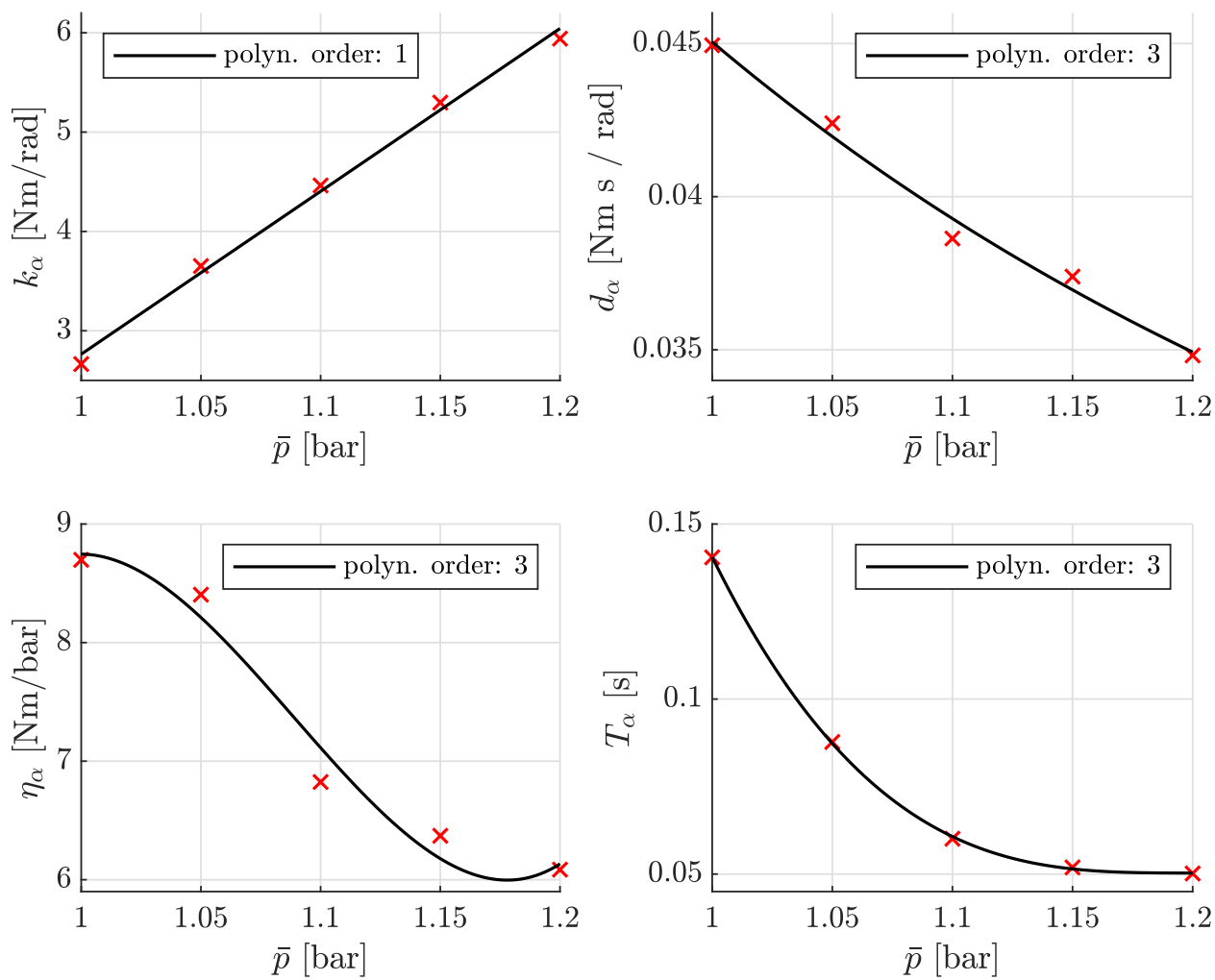

Figure 11. The parameters of the transfer function, $G_{\alpha}(s, \bar{p}, m)$, as a function of the lower pressure level $\bar{p}$. The red crosses indicate the parameter values from the identification experiments and the black solid lines show the first or third order polynomial fits. The stiffness parameter (top left plot) shows a linear relation with the lower pressure level, $\bar{p}$.
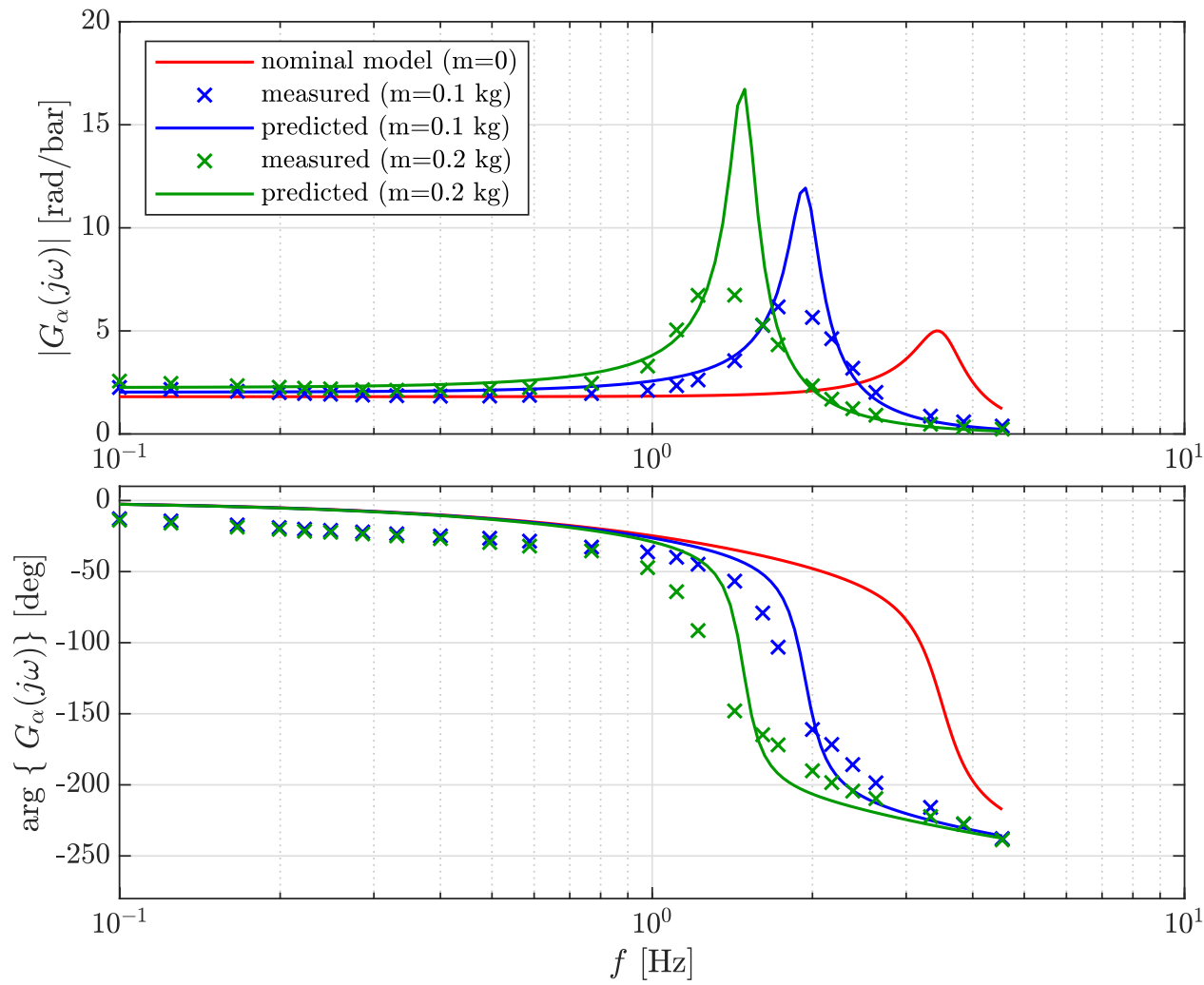

Figure 12. The figure shows a comparison of the frequency response when a payload mass is attached. The solid red line shows the (fitted) frequency response where no mass is attached and is referred 
to as the nominal model. The solid blue and green lines show the predicted frequency responses based on the nominal model and extrapolating the effect of the mass based on (11). The blue and green crosses indicate the measured frequency response with an attached payload mass of $0.1 \mathrm{~kg}$ or $0.2 \mathrm{~kg}$, respectively. The lower pressure level is set to 1.1 bar for both experiments. The left shift of the resonance frequency is accurately predicted by the model, while the rise of resonance is slightly overestimated.

\subsection{Model Limitations}

While the linear parameter varying model captures the principle dynamics of the system, there are neglected dynamic effects that can, e.g., be attributed to the viscoelastic material behavior as discussed in [5]. An example of slowly changing viscoelastic material behavior is shown in Figure 13. The stress relaxation happening after the inflation of each actuator for a certain time, causes a shift in the angular response in the $\alpha$ - $\beta$-plane. This effect happens at a slower timescale compared to the identification experiments shown in Figure 10 and potentially depends on the history of past inputs and states. Feedback control is indispensable to compensate for such effects. Alternatively, as the behavior is repeatable for each experiment, the application of learning control is a viable alternative, as discussed in [48].

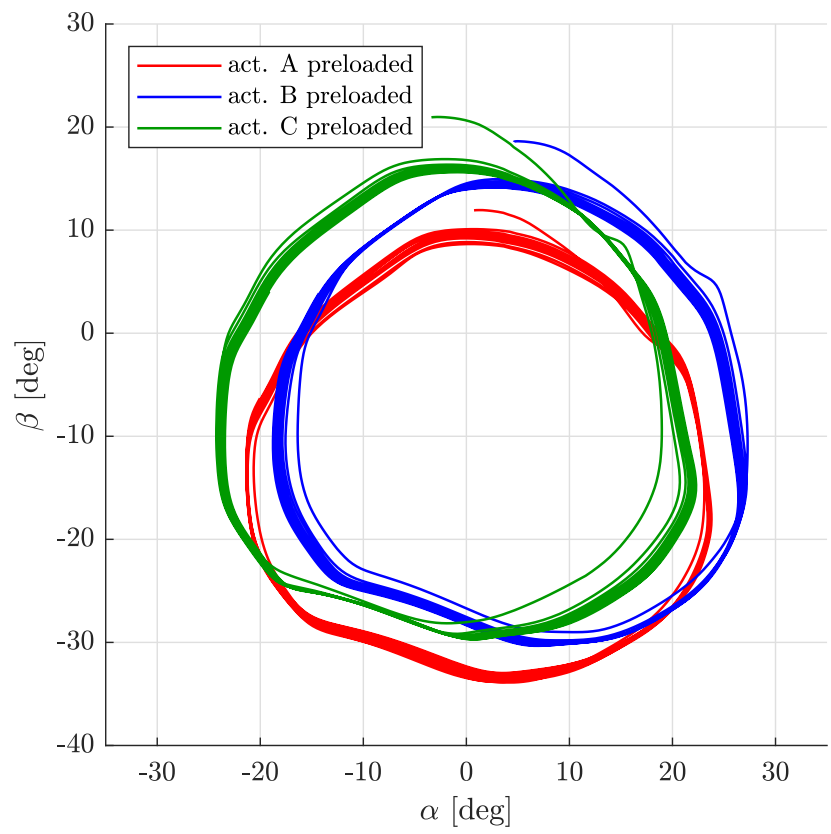

Figure 13. The figure shows the angular response for each of the three experiments in which one of the three actuators is preloaded, i.e., inflated to 1.3 bar for $5 \mathrm{~min}$. Then, sinusoidal set point trajectories for $\Delta p_{\alpha, \mathrm{SP}}$ and $\Delta p_{\beta, \mathrm{SP}}$ are commanded, resulting in a circle in the $\alpha$ - $\beta$-plane. Thereby, $\bar{p}$ is set to ambient pressure. The procedure is repeated for actuators $B$ and $C$ being preloaded and the same pressure setpoint trajectories are commanded. The recorded circles are shifted away from the particular actuator that was preloaded. Within each experiment, the single realizations of the circle show little variation, emphasizing the good repeatability achievable with the system.

\subsection{Longitudinal Movement}

The control allocation strategy presented in Section 4.2 decouples the two rotational degrees of freedom from the lower pressure level (or joint stiffness). However, a change in the lower pressure level can cause a disturbance on the angles. The reason for this behavior is that the maximum attainable angle range for fixed values of $\Delta p_{\alpha}$ and $\Delta p_{\beta}$ decreases as the lower pressure level increases. The behavior can be seen in the right plot of Figure 8 with the extent of the figure-eight trajectory decreasing for increasing values of $\bar{p}$. The 
intuition is that the robotic arm is pushed towards a straight configuration when all three actuators are inflated. The induced error in the $\alpha$ and $\beta$-directions can approximately be described by the following model,

$$
\begin{aligned}
& e_{\alpha}=c_{1} \alpha_{\mathrm{SP}} \bar{p} \\
& e_{\beta}=c_{1} \beta_{\mathrm{SP}} \bar{p},
\end{aligned}
$$

where $c_{1}>0$ is a conversion factor from rad bar to rad. The error depends on the sign and the magnitude of the current setpoint angle. For a larger setpoint angle, an increase in $\bar{p}$ causes a larger error. The model is exploited in the feed forward control strategy discussed in Section 5.3.

\section{Control}

The control strategy for the robotic arm is presented in this section. First, the available sensory feedback is discussed followed by the cascaded control architecture implemented. The low level feedback control approaches for the pressure dynamics are discussed, followed by the controller for the arm dynamics leveraging the model presented in the previous section. Two feed forward compensation schemes are presented that compensate for the effect of gravity and the longitudinal movement discussed in Section 4.5.

\subsection{Sensory Feedback}

The motion capture system provides sensory feedback of the Cartesian coordinates $(x, y, z)$ of the markers attached to the tip of the movable link. The two angles $\alpha$ and $\beta$ are obtained by applying the following formulas,

$$
\begin{aligned}
& \alpha=\arcsin \left(-\left(y-y_{0}\right) / R\right) \\
& \beta=\arcsin \left(\left(x-x_{0}\right) /(R \cos (\alpha))\right),
\end{aligned}
$$

where $R$ denotes the length from the pivot point to the markers and $\left(x_{0}, y_{0}\right)$ the Cartesian coordinates of the pivot point. All three parameters are determined from a least squares regression applied to data recorded during a calibration procedure where the robotic arm is moved while recording the tip coordinates. The longitudinal elongation is not explicitly computed, since it is controlled in a feed forward manner. Sensory feedback of the actuator pressures is provided by three pressure sensors.

\subsection{Feedback Control}

A cascaded control architecture is implemented that is based on a timescale separation between the faster pressure dynamics and the slower arm dynamics. Hence, the pressure dynamics of each actuator are controlled in independent inner control loops running at $1000 \mathrm{~Hz}$ and relying on sensory feedback from the pressure sensors. The arm dynamics are controlled in an outer control loop running at $50 \mathrm{~Hz}$ and using sensory feedback from the angles $\alpha$ and $\beta$. The pressure setpoints, $\left(p_{\mathrm{A}, \mathrm{SP}}, p_{\mathrm{B}, \mathrm{SP}}, p_{\mathrm{C}, \mathrm{SP}}\right)$, are computed by the position controller in the outer loop and form the interface to the inner loops of the cascaded control architecture.

A cascaded control architecture allows for the use of all the available sensory feedback at its full rate and the handling of non-linearities of the pressure dynamics in separate inner loops. As a consequence, the derivation of the outer control loop is simplified and the position controller can be tuned more aggressively. The block diagram is shown in Figure 14. 


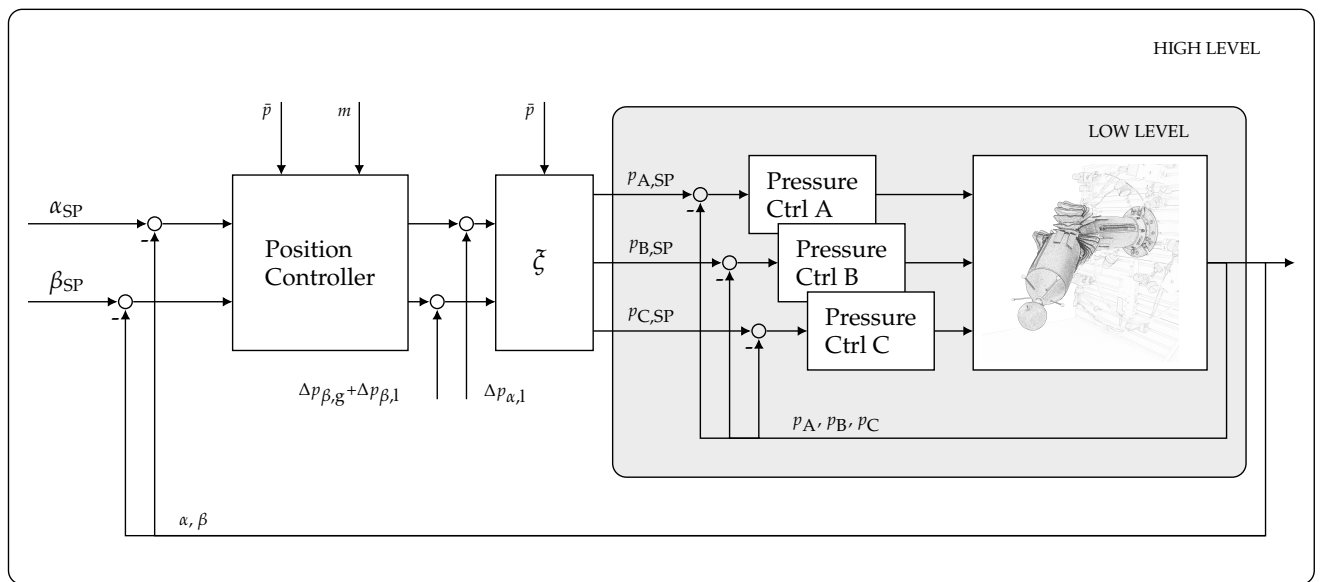

Figure 14. The cascaded control architecture employed for the soft robotic arm. The architecture is structured into a low level part that is executed at $1000 \mathrm{~Hz}$ on an embedded hardware and a high level part that is executed on a laptop computer at $50 \mathrm{~Hz}$. A timescale separation between the pressure and arm dynamics is exploited, where each actuator pressure is controlled by a proportional-integralderivative controller in a separate inner control loop. The position controller in the outer loop uses sensory feedback of the angles to compute two virtual control inputs $\Delta p_{\alpha, \mathrm{SP}}$ and $\Delta p_{\beta, \mathrm{SP}}$. The gain scheduled position controller depends on the commanded value for $\bar{p}$ and the known payload mass $m$. The control allocation strategy is applied to map the virtual control inputs to the actuator pressure set points that are the reference signals for the inner loops. Feed forward control action is added to compensate for the effect of gravity and a longitudinal actuation of the arm.

Three separate proportional-integral-derivative controllers (PID controller) are used to control the inner loops,

$$
\begin{aligned}
& u_{A}=K_{\mathrm{P}}\left(p_{\mathrm{A}, \mathrm{SP}}-p_{\mathrm{A}}\right)+K_{\mathrm{I}} \int\left(p_{\mathrm{A}, \mathrm{SP}}-p_{\mathrm{A}}\right) d t+K_{\mathrm{D}} \frac{d}{d t} p_{\mathrm{A}} \\
& u_{B}=K_{\mathrm{P}}\left(p_{\mathrm{B}, \mathrm{SP}}-p_{\mathrm{B}}\right)+K_{\mathrm{I}} \int\left(p_{\mathrm{B}, \mathrm{SP}}-p_{\mathrm{B}}\right) d t+K_{\mathrm{D}} \frac{d}{d t} p_{\mathrm{B}} \\
& u_{\mathrm{C}}=K_{\mathrm{P}}\left(p_{\mathrm{C}, \mathrm{SP}}-p_{\mathrm{C}}\right)+K_{\mathrm{I}} \int\left(p_{\mathrm{C}, \mathrm{SP}}-p_{\mathrm{C}}\right) d t+K_{\mathrm{D}} \frac{d}{d t} p_{\mathrm{C}}
\end{aligned}
$$

with $u_{A}, u_{B}$, and $u_{C}$ denoting the input to the valves. The integrator is limited to avoid excessive control action for infeasible setpoints.

The arm dynamics are controlled in an outer loop by a position controller that leverages the control allocation strategy presented in Section 4.2. Based on the current error in both angles $\alpha$ and $\beta$, the decoupled position controller computes separate correction inputs, $\Delta p_{\alpha, \mathrm{SP}}$ and $\Delta p_{\beta, \mathrm{SP}}$. The controller is based on the model identified in Section 4.3 and relies on a pole-zero cancellation,

$$
C_{\alpha}(s, \bar{p}, m)=\kappa_{\alpha} \frac{(m+M / 4) R_{0}^{2} s^{2}+d_{\alpha}(\bar{p}) s+k_{\alpha}(\bar{p})}{s},
$$

and analogously for $\beta$. The gain of the controller is denoted by $\kappa_{\alpha}$. The linear gainscheduled feedback controller compensates for the effect of the payload mass, $m$, and the lower pressure level, $\bar{p}$. Thereby, the parameters $\bar{p}$ and $m$ are assumed to vary sufficiently slowly with respect to time (p. 48 in [56]). A case where this assumption is not fulfilled will be discussed in Section 5.3. The order of the feedback controller (15) corresponds to a PID controller, where the ratio between the gains is parameterized by the identified model. The computed control inputs, $\Delta p_{\alpha, \mathrm{SP}}$ and $\Delta p_{\beta, \mathrm{SP}}$, are then converted to the three actuator pressure setpoints by applying (6).

As a consequence of leveraging the control allocation strategy, the feedback control action does not affect the lower pressure level and therefore, does not change the joint 
stiffness. Hence, the compliance of the system is preserved and can be commanded independently of the feedback controller. Therefore, a typical concern in the control of soft robots that accurate feedback control comes at the cost of a reduction of the compliance of the system (see [34]) can be prevented.

It remains to verify that the closed-loop system is stable. Therefore, the complementary sensitivity function is calculated for $\alpha$,

$$
\begin{aligned}
T_{\alpha}(s, \bar{p}) & =\frac{C_{\alpha}(s, \bar{p}, m) \cdot G_{\alpha}(s, \bar{p}, m)}{1+C_{\alpha}(s, \bar{p}, m) \cdot G_{\alpha}(s, \bar{p}, m)} \\
& =\frac{\frac{\kappa_{\alpha} \eta_{\alpha}(\bar{p})}{T_{\alpha}(\bar{p})}}{s^{2}+\frac{1}{T_{\alpha}(\bar{p})} s+\frac{\kappa_{\alpha} \eta_{\alpha}(\bar{p})}{T_{\alpha}(\bar{p})}},
\end{aligned}
$$

and accordingly for $\beta$. Thereby, the payload mass is assumed to be known exactly and as a consequence, its effect on the dynamics is compensated by the controller. There is a dependency of $T_{\alpha}(s, \bar{p})$ on $\bar{p}$ due to the first term in (11) related to the actuator dynamics that are not compensated by the controller. The closed loop system for $\alpha$ (and similarly for $\beta$ ) is a second order system and according to the Routh-Hurwitz criterion (see [57]) is stable, as the coefficients of the denominator polynomial are strictly positive for $\kappa_{\alpha, \beta}>0$ and the parameters identified.

In the following paragraph, we verify the assumption of a timescale separation between the pressure and arm dynamics that form the basis for the cascaded control approach. First, the effect of different magnitudes of timescale separation on the resulting closed loop behavior is investigated. To this end, the following simplified system is considered with the inner control loop as,

$$
\ddot{x}=\dot{x}_{\mathrm{d}}-\dot{x},
$$

where $x$ is the state, $\dot{x}_{\mathrm{d}}$ the desired velocity. The normalized first order system has a time constant of one. The outer control loop is assumed to be,

$$
\dot{x}_{\mathrm{d}}=\frac{1}{\sigma}\left(x_{\mathrm{r}}-x\right),
$$

where $x_{\mathrm{r}}$ is the reference for $x$ and $\sigma$ denotes the time constant of the first order system. Combining the inner and outer loops yields the following second order system,

$$
\ddot{x}=\frac{1}{\sigma}\left(x_{\mathrm{r}}-x\right)-\dot{x} \Longleftrightarrow \ddot{x}+\dot{x}+\frac{1}{\sigma} x=\frac{1}{\sigma} x_{\mathrm{r}} .
$$

Note that (16) also corresponds to a second order system. The poles of (19) are given by,

$$
s^{2}+s+\frac{1}{\sigma}=0 \Rightarrow s_{1,2}=\frac{-1 \pm \sqrt{1-4 / \sigma}}{2} .
$$

We consider four different values of $\sigma$ and investigate the resulting closed loop behavior in Table 2. 
Table 2. The closed loop poles for a second order system, where the ratio of the time constants of the outer and inner loop is $\sigma$. For $\sigma \rightarrow \infty$, we have a perfect timescale separation with the closed loop poles equal to the poles of the inner loop $\left(s_{1}=-1\right)$ and the outer loop $\left(s_{2}=0\right)$. For $\sigma=10$ (the outer loop is ten times slower than the inner loop), we still obtain a reasonably good timescale separation. For $\sigma=4$ the two poles coincide (corresponding to critical damping) and for values of $\sigma<4$, e.g., $\sigma=2$, the poles become complex conjugated, resulting in a underdamped system. For the last case, the assumption of a timescale separation is clearly violated, resulting in the introduction of oscillatory behavior.

\begin{tabular}{ccc}
\hline$\sigma$ & $s_{\mathbf{1}}$ & $\boldsymbol{s}_{\mathbf{2}}$ \\
\hline$\infty$ & -1 & 0 \\
10 & -0.88 & -0.11 \\
4 & -0.5 & -0.5 \\
2 & $-0.5+0.5 i$ & $-0.5-0.5 i$ \\
\hline
\end{tabular}

If the time constant of the outer loop is ten times slower than the time constant of the inner loop, the assumption of a timescale separation is fulfilled and a cascaded control architecture is justified without the risk of oscillatory behavior being introduced.

In the next step, we experimentally investigate the time constant of the inner pressure control loop. Since the pressure dynamics strongly depend on the available flow capacity of the valves employed (see Section 3.3) the analysis is performed for different valve flow capacities, where the flow capacity is limited to $50 \%$ or $25 \%$ of its nominal value in software. The closed-loop pressure dynamics are identified from a series of pressure steps in actuator A. Both positive and negative steps of magnitude 0.1 bar are considered in the range between 1.0 bar and 1.5 bar. A linear first order model is identified based on this data, which predicts the behavior relatively accurately. The magnitude of the frequency response for the different flow capacities is shown in Figure 15. It can clearly be seen that using valves with a sufficient flow capacity is crucial to ensure a sufficiently small time constant of the closed loop pressure dynamics.

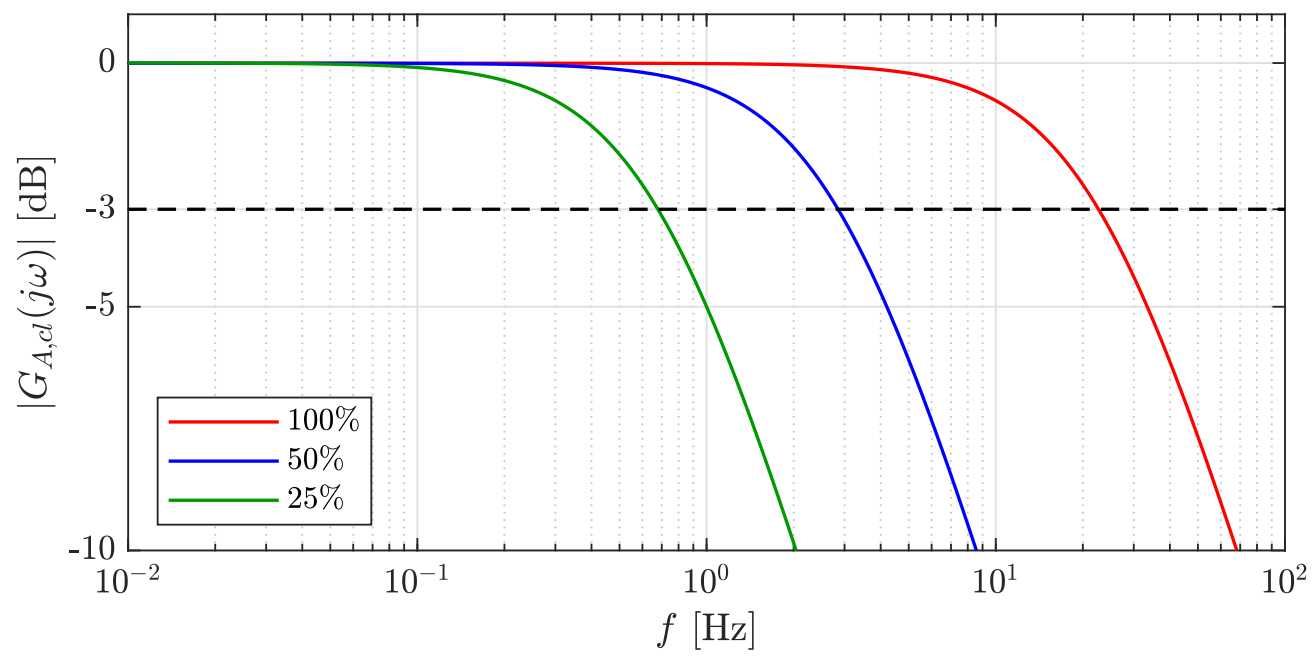

Figure 15. The identified closed-loop pressure dynamics for actuator A resulting from a series of pressure steps. The red curve shows the magnitude of the frequency response when the full flow capacity of the proportional valve is used $(100 \%)$. The blue and green curves show the magnitude when the flow capacity is limited to $50 \%$ and $25 \%$ of the nominal value, respectively. The black dashed line indicates the $-3 \mathrm{~dB}$ line. The cutoff frequencies decrease from $22.7 \mathrm{~Hz}$ for the red curve to $2.9 \mathrm{~Hz}$ for the blue curve and $0.7 \mathrm{~Hz}$ for the green curve.

Next, we investigate the time constant of the arm dynamics. Therefore, we compute the cutoff frequency (gain equal to $-3 \mathrm{~dB}$ ) of the complementary sensitivity function (16), 
for both $\alpha$ and $\beta$ and different values of $\bar{p}$, in the range of 1.0 bar to 1.2 bar. The resulting cutoff frequencies lie in the range of $1.1 \mathrm{~Hz}$ to $1.7 \mathrm{~Hz}$.

In comparison to the cutoff frequency of the closed-loop pressure dynamics $(22.7 \mathrm{~Hz}$ when using the full flow capacity of the valve), we can conclude that the cutoff frequencies (and similarly the time constants) of the closed loop arm dynamics are separated by more than a factor of ten. It follows from the behavior analyzed in Table 2 that no oscillatory behavior is introduced by the cascaded control approach and a violation of the underlying timescale separation assumption.

This section is concluded by an experimental evaluation of the feedback control approach presented. Thereby, the ability to independently adjust the joint stiffness and to control the position of the arm is validated. The same reference trajectory for $\alpha$ and $\beta$ is tracked for different levels of joint stiffness and for the case of a payload mass, attached or not. The results are shown in Figure 16. The results indicate the ability of the controller to adjust the joint stiffness and independently control the rotational degrees of freedom with and without a payload mass attached. Furthermore, the results show that the dynamical effects of changing joint stiffness or an attached payload mass are sufficiently compensated by the controller proposed, leading to a similar angular response for the different cases considered. However, a mismatch between true and commanded payload mass yields a clearly degraded tracking performance as we expected. This emphasizes the importance of systematically addressing the effect of an attached payload mass in the feedback controller as a result of its strong influence on the dynamics.

\subsection{Feed Forward Control}

In this section, two feed forward compensations are presented to compensate for known disturbances. A first feed forward component is added to compensate for gravitational effects acting on $\beta$ (see Figure 7),

$$
\Delta p_{\beta, \mathrm{g}}=\frac{R_{0 g}}{\eta_{\beta}}\left(\frac{M}{2}+m\right) \cos \left(\beta_{S P}\right),
$$

where $\eta_{\beta}$ is the conversion gain from pressure to torque (see (9)).

The second feed forward control action addresses a disturbance caused by a fast change in $\bar{p}$. In general, the lower stiffness level is changed sufficiently slowly, such that any effect on the angles as described in Section 4.5 can be compensated by the feedback controller. However, when $\bar{p}$ is increased quickly to cause a longitudinal elongation for grasping an object, the disturbance can not be rejected sufficiently quickly by the feedback controller. Therefore, the following feed forward control action is applied based on the model (12),

$$
\begin{aligned}
& \Delta p_{\alpha, 1}=\lambda \frac{k_{\alpha}}{\eta_{\alpha}} c_{1} \alpha_{\mathrm{SP}} \max \left(\bar{p}-\bar{p}_{\min }, 0\right) \\
& \Delta p_{\beta, 1}=\lambda \frac{k_{\beta}}{\eta_{\beta}} c_{1} \beta_{\mathrm{SP}} \max \left(\bar{p}-\bar{p}_{\min }, 0\right),
\end{aligned}
$$

where $k_{\alpha} / \eta_{\alpha}$ is the steady state conversion from angle to pressure setpoint (compare (11)). The parameter $\lambda$ is a gain tuned for best performance, with a value of $\lambda=1.3$ yielding the best results. The parameter, $\bar{p}_{\min }$, is a lower bound for $\bar{p}$ from when the feed forward action is used. A comparison when $\bar{p}$ is rapidly changed and the feed forward control action is used or not used is shown in Figure 17. The proposed feed forward scheme reduces the maximum error by a factor of two. 


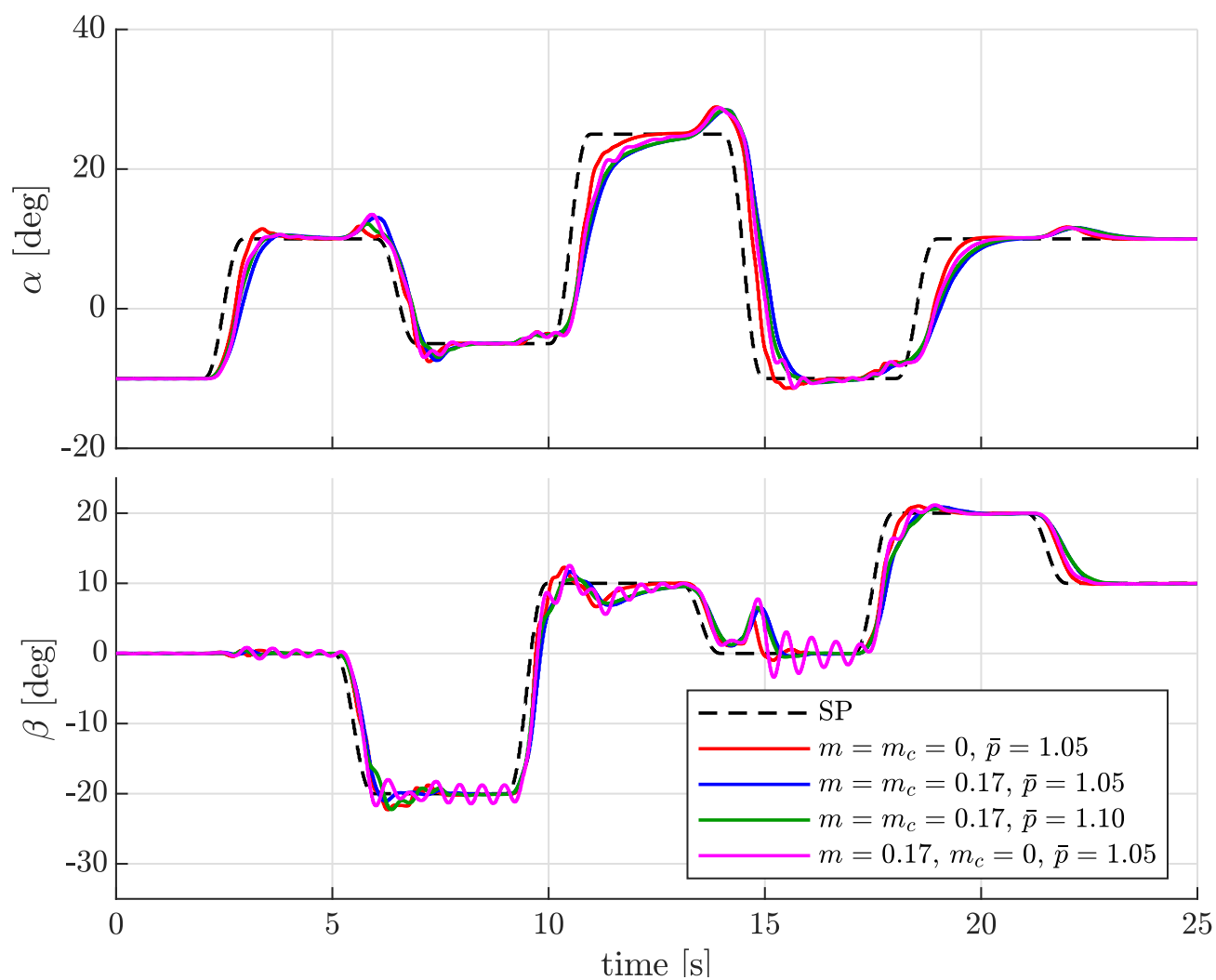

Figure 16. The resulting tracking performance when relying on the feedback controller is shown in the top plot for $\alpha$ and in the bottom plot for $\beta$. The black dashed lines denote the set point trajectories and the colored lines the four cases investigated. Thereby, the following parameters are changed: the attached payload mass, $m$, the value set for the payload mass in the controller, $m_{c}$, and the lower pressure level $\bar{p}$. Normally, the two values for $m$ and $m_{\mathrm{c}}$ are identical, but for the sake of this investigation, we also consider differing values. The red line shows the results when no payload mass is attached and the joint stiffness is set to the lower level ( $\bar{p}=1.05$ bar $)$ and the blue line shows the case where a payload mass is attached and the joint stiffness is set to a low level. The green line represents the case where a payload mass is attached and the joint stiffness is set to the high level $(\bar{p}=1.10$ bar $)$. For all three cases, the controller had knowledge of the true payload mass attached $\left(m_{c}=m\right)$, resulting in similar tracking performance for both $\alpha$ and $\beta$. The purple line indicates the behavior when a payload mass is attached $(m=0.17 \mathrm{~kg})$, but the controller assumes no payload mass $\left(m_{c}=0\right)$. The joint stiffness is set to the lower level for this case. Distinct oscillations are visible for the last case, when considering $\beta$. As a consequence of the mismatch between the true mass and the value commanded to the controller, the control performance is degraded. Additionally, note the slight errors occurring in one angle, when commanding a change in the other angle and vice versa. 


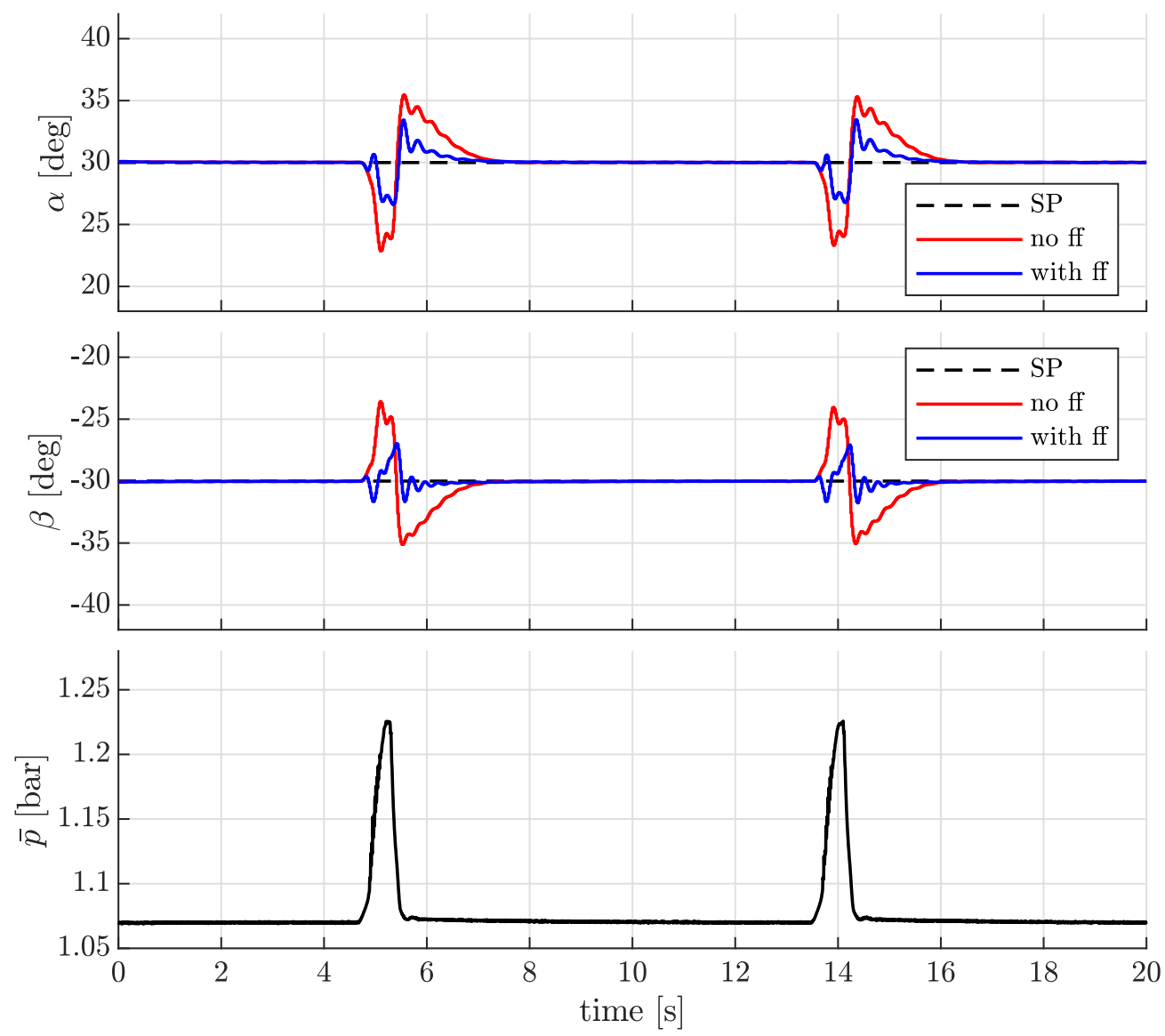

Figure 17. The lower pressure level, $\bar{p}$, as shown in the bottom plot, is rapidly increased to cause a longitudinal elongation for grasping an object. The top and middle plots show a comparison of the angles $\alpha$ and $\beta$ when no feed forward (ff) control action is used (red curve) and when the proposed feed forward strategy is employed (blue curve). Relying on the feed forward approach reduces the maximum error in $\alpha$ from $7.1 \mathrm{deg}$ to $3.5 \mathrm{deg}$ and in $\beta$ from $6.4 \mathrm{deg}$ to $3.0 \mathrm{deg}$. In the case where no feed forward control action is used, we purely rely on the feedback controller. Note that an increase in $\bar{p}$ causes the robotic arm to be deflected towards the origin and vice versa for a decrease in $\bar{p}$.

\section{Applications}

In this section, we demonstrate two object manipulation applications with the soft robotic arm. In the first example, a rubber ball is picked up at a known location and placed at another know location. In the second example, the rubber ball is picked up from the same initial location, but handed over to a human (for an example of a different object being manipulated, we refer to [48]). Due to the feed forward strategy developed and discussed in Section 5.3, the grasping of the object is possible without the application of the learning control scheme that was required in [48] to reliably grasp an object and drop it into a box. Here, the object being manipulated is a rubber ball with a diameter of $70 \mathrm{~mm}$ and a mass of $0.16 \mathrm{~kg}$. The object is placed on a wooden platform with a circular cutout to hold it in place. For both applications a flexible suction cup (FSGA 33 NK-45 G1/4-AG from Schmalz) is used that has an additional bellow and can adapt to the object being manipulated. The reader is referred to the video attachment for an illustration of the experiments conducted (see Supplementary Video S1).

\subsection{Pick and Place Application}

The pick and place application consists of the following phases: First, the robotic arm moves from the idle location to the known pickup location. The lower actuator pressure level is temporarily increased to longitudinally elongate the movable link. Activating the vacuum enables the robotic arm to grasp the object with the suction cup. Then, the robotic 
arm moves the object to the target location. Finally the object is placed by releasing the vacuum and quickly activating the ejection impulse. Throughout the application, the mass is assumed to be known, and is adjusted during the different phases of the application depending on whether the robotic arm is in contact with the object or not. The significance of this assumption could be reduced by using an average mass and detuning the controller to robustly work with a wider range of payload masses. The controller gains are set to $\kappa_{\alpha}=\kappa_{\beta}=0.9$. The lower actuator pressure level is set to a constant value of 1.04 bar, except when the robotic arm longitudinally extends to grasp the object. A visualization of the application is provided in Figure 18. The robotic arm can reliably move the rubber ball from the initial location to the target location. The longitudinal elongation of the arm, the lifting and the placing of the ball cause a disturbance that the robotic arm is able to reject.

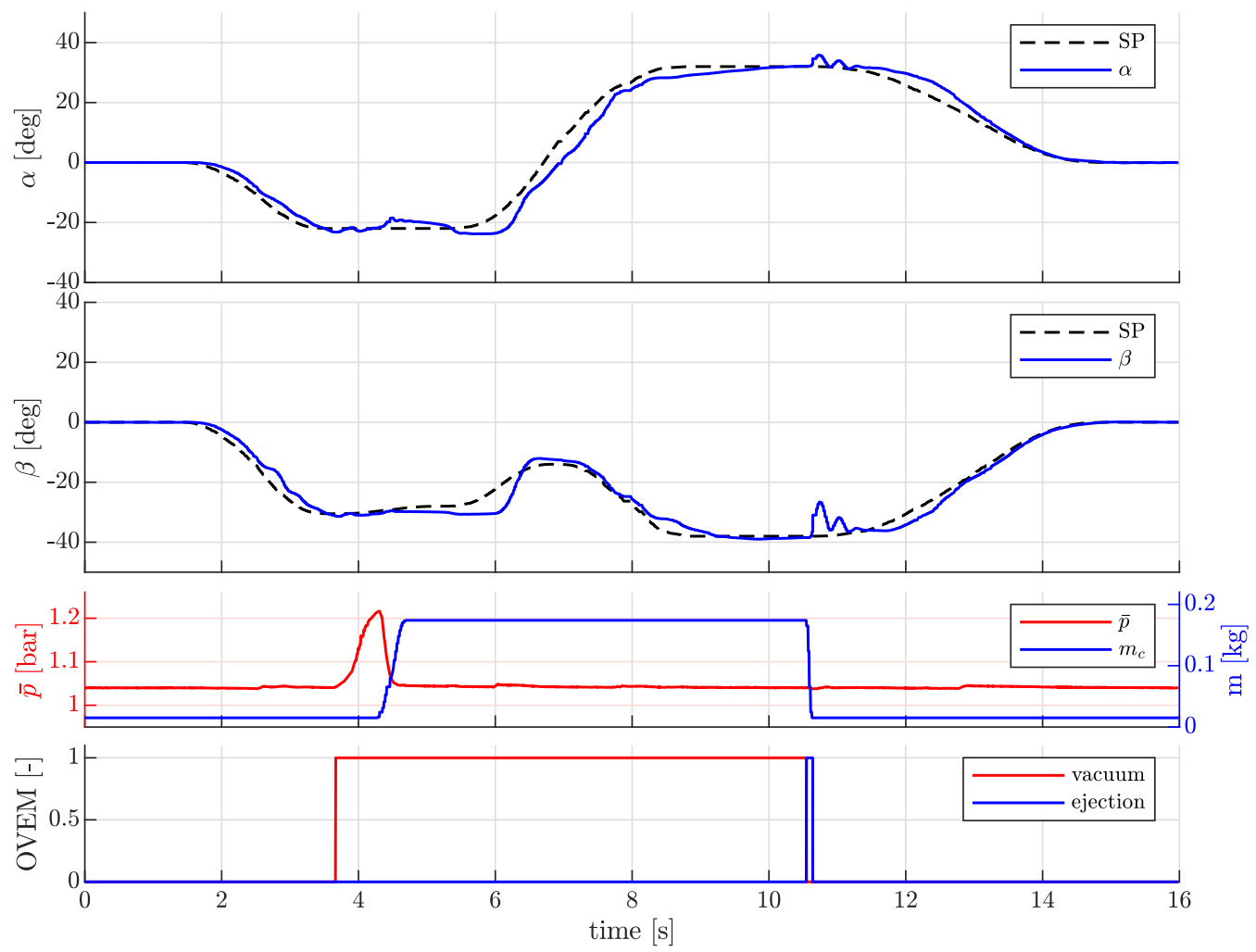

Figure 18. A visualization of the pick and place application: The top two plots show the angles $\alpha$ and $\beta$ and their setpoints, respectively. The arm starts from the idle position, $(\alpha, \beta)=\left(0^{\circ}, 0^{\circ}\right)$, and picks up the object at the location, $\left(-22^{\circ},-30.5^{\circ}\right)$, moves it to the target location at $\left(-32^{\circ},-38^{\circ}\right)$, and then moves back to the idle location $\left(0^{\circ}, 0^{\circ}\right)$. The arm is raised in the positive $\beta$-direction between picking the object and releasing it, to avoid interfering with the platforms where the object is picked from and released to. Releasing the object causes an error in both angles due to the sudden change of mass. The third plot shows the lower actuator pressure level, $\bar{p}$, and the load mass assumed by the controller. When the suction cup points towards the object, the lower actuator pressure level is increased to cause a longitudinal elongation and pick up the object. The payload mass commanded to the controller is continuously increased from the weight of the suction cup to the combined weight of suction cup and manipulated object. When the object is released, the commanded mass is continuously decreased back to the weight of the suction cup. The bottom plot shows the control inputs of the vacuum generation unit. The red curve shows the vacuum input to generate a vacuum at the suction cup. It is activated shortly before the suction cup touches the object to ensure a reliable picking procedure. The blue curve shows the ejection impulse used to release the vacuum when placing the object. 


\subsection{Collaborative Application with Human}

In the second application, we demonstrate that the soft robotic arm can pick up an object and hand it over to a human. Thereby, the safety aspect discussed in Section 2 becomes apparent as the collaborative application requires close contact between the robot arm and the human. Once the robot arm has moved the object to the target location, it needs to determine when it should hand the object over to the human. Therefore, a property of the soft robotic arm is leveraged: The compliance of the joint allows the human to move the object and thereby also the robotic arm that still grasps the rubber ball. Once the angular error between the current robot position and its setpoint exceeds a predefined threshold, the robot arm releases the object. The application is visualized in Figure 19. Both applications can be conducted at an increased execution speed by applying the iterative learning control scheme presented in [48]. This allows for compensation of repetitive disturbances arising from aggressive movements and ensures accurate tracking performance. For the sake of brevity, the learning control scheme is not presented here and the reader is referred to [48]. A visualization of the collaborative application with and without applying the learning scheme is provided in the video attachment (see Supplementary Materials).

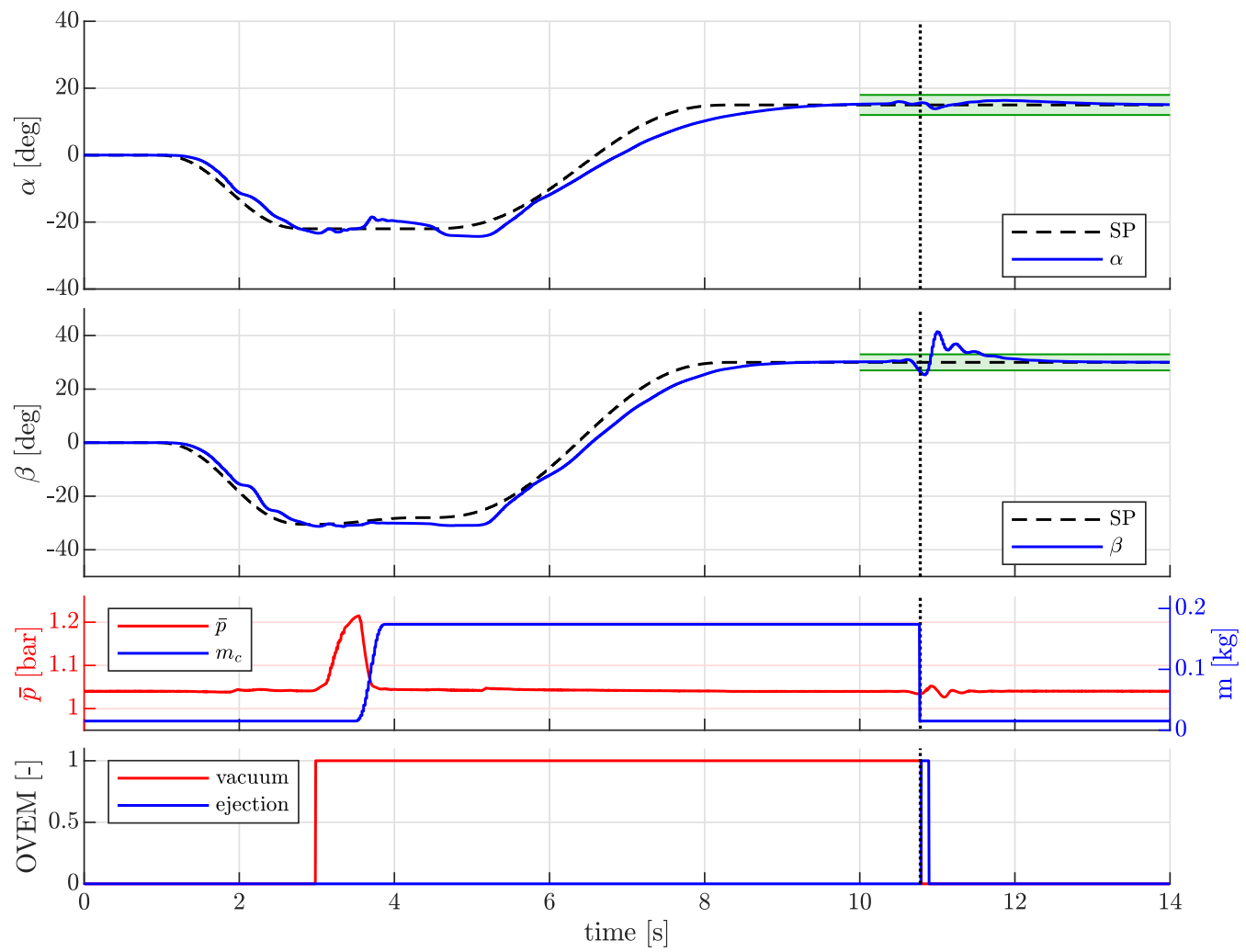

Figure 19. Visualization of the collaborative application where the robot picks up an object and hands it over to a human: The top two plots show the angles $\alpha$ and $\beta$ and their setpoints, respectively. The third plot shows the lower actuator pressure level and the assumed payload mass. The bottom plot indicates the control inputs of the vacuum generation unit. The arm starts from the idle position, $(\alpha, \beta)=\left(0^{\circ}, 0^{\circ}\right)$, picks up the object at the initial location, $\left(-22^{\circ},-30.5^{\circ}\right)$, moves it to the final position at $\left(15^{\circ}, 30^{\circ}\right)$, and waits for the human interaction. The robot arm maintains the vacuum as long as the object lies in the green region corresponds to an angular range of $\pm 3^{\circ}$ of the setpoint in $\alpha$ or $\beta$, respectively. As soon as the human moves the object outside the green region (the time instance is indicated by the vertical, dotted black line), the payload mass assumed by the controller is adjusted, the vacuum is released and the ejection impulse is activated for $0.1 \mathrm{~s}$ to purge the vacuum. A human interaction is only expected for $t \geq 10 \mathrm{~s}$ to exclude a triggering of the release condition due to transient tracking errors. 


\section{Conclusions}

This article presents an inflatable robotic arm for object manipulation. The design is motivated by a first principle safety analysis and realized by state-of-the-art manufacturing techniques, leading to a robust and reliable system. The mechanical, pneumatic, and electronic subsystems are discussed in detail. A property of the control allocation strategy employed is discussed, along with its implication for the use of the valves. The cascaded control architecture, which is commonly applied in soft robotics, relies on the assumption of a timescale separation between the faster pressure dynamics and the slower arm dynamics. The closed loop pressure dynamics are identified for different valve flow capacities and it is shown that an insufficient valve flow capacity prevents a timescale separation, and can lead to oscillatory behavior and a degradation of the overall control performance. The analysis conducted presents a tool that can be applied during the design process of a pneumatically actuated soft robot for determining the optimal valve size. First principles can be used to estimate the closed loop pressure and arm dynamics and the resulting time constants.

A feed forward compensation scheme is derived that mitigates errors induced by a short-term increase of all actuator pressures to cause a longitudinal elongation for grasping an object. Two pick and place applications are realized with the causal feedback strategy. Thereby, no learning control is required, as was the case in previous work. Compared to [48], where the object was simply dropped into a box, a special focus lies on the placing part of the application. In the first example, the object is placed at a known target location and in the second example it is handed over to a human. Thereby, the compliance of the soft robotic system is leveraged for detecting the interaction with the human and releasing the object. A previously developed iterative learning control approach was applied and the results are presented in the video attachment. This allows an increase in the execution speed while maintaining sufficient tracking performance.

An interesting direction for future work is the extension of the modeling approach presented to describe dynamic effects happening at slower timescales that are attributed to the viscoelastic material behavior. Techniques from disturbance estimation could be used to identify the current offsets, to compensate for them, and consequently to lower the amount of integral control action required.

Although two applications could be realized with the system presented, the number of degrees of freedom could be limiting for more complex applications. An idea to circumvent this limitation would be to connect the inflatable robotic arm to a conventionally actuated base (e.g., using electric motors). This would increase the number of degrees of freedom, but also ensure that all parts sticking out from the base are soft and lightweight and, therefore, preserve the safety considerations.

The interaction detection presented in this work is based on the compliance of the robotic arm and a measured positional deviation. An interesting extension would be the estimation of the interaction forces based on the model presented. This would also be interesting to further improve the safety of the robotic arm by limiting the forces exerted.

Finally, there are many interesting applications beyond the scope of pick and place applications that are subjects for future work. One example is joint object manipulation together with a human, where the capability of adjusting the joint stiffness is promising.

Supplementary Materials: The following files are available online https:/ / www.mdpi.com/article/ 10.3390/act10110299/s1. S1: A Video of the experiments conducted. S2: The schematics of the custom interface board. S3: The derivation of the control allocation strategy.

Author Contributions: Conceptualization, M.H., J.Z. and R.D.; Methodology, M.H., J.Z. and R.D.; Software, M.H. and J.Z.; Validation, M.H. and J.Z.; Formal Analysis, M.H. and J.Z.; Investigation, M.H. and J.Z.; Resources, M.H. and R.D.; Data Curation, M.H. and J.Z.; Writing-Original Draft Preparation, M.H.; Writing-Review and Editing, M.H., J.Z. and R.D.; Visualization, M.H.; Supervision, M.H. and R.D.; Project Administration, M.H. and R.D.; Funding Acquisition, M.H. and R.D. All authors have read and agreed to the published version of the manuscript.

Funding: This research received no external funding. 
Institutional Review Board Statement: Not applicable.

Informed Consent Statement: Not applicable.

Data Availability Statement: Not applicable.

Acknowledgments: The authors would like to thank Matthias Müller, Helen Hanimann, Michael Egli, and Daniel Wagner for their support during the development of the system. Special thanks goes to Björn Walser from Walser Kunstofftechnik AG, the technical support of Festo Schweiz AG, Marcel Gnos from BIBUS Schweiz AG and Ulrich Lemann and Benjamin Geisser from Wyss Zurich.

Conflicts of Interest: The authors declare no conflict of interest.

\section{Appendix A. System Identification}

In this section, the plots of the system identification experiments performed in $\beta$ direction are presented. The measured and fitted frequency response for $G_{\beta}(s, \bar{p}, m)$ is shown in Figure A1. The parameters of $G_{\beta}(s, \bar{p}, m)$ as a function of $\bar{p}$ and their corresponding fits are shown in Figure A2.
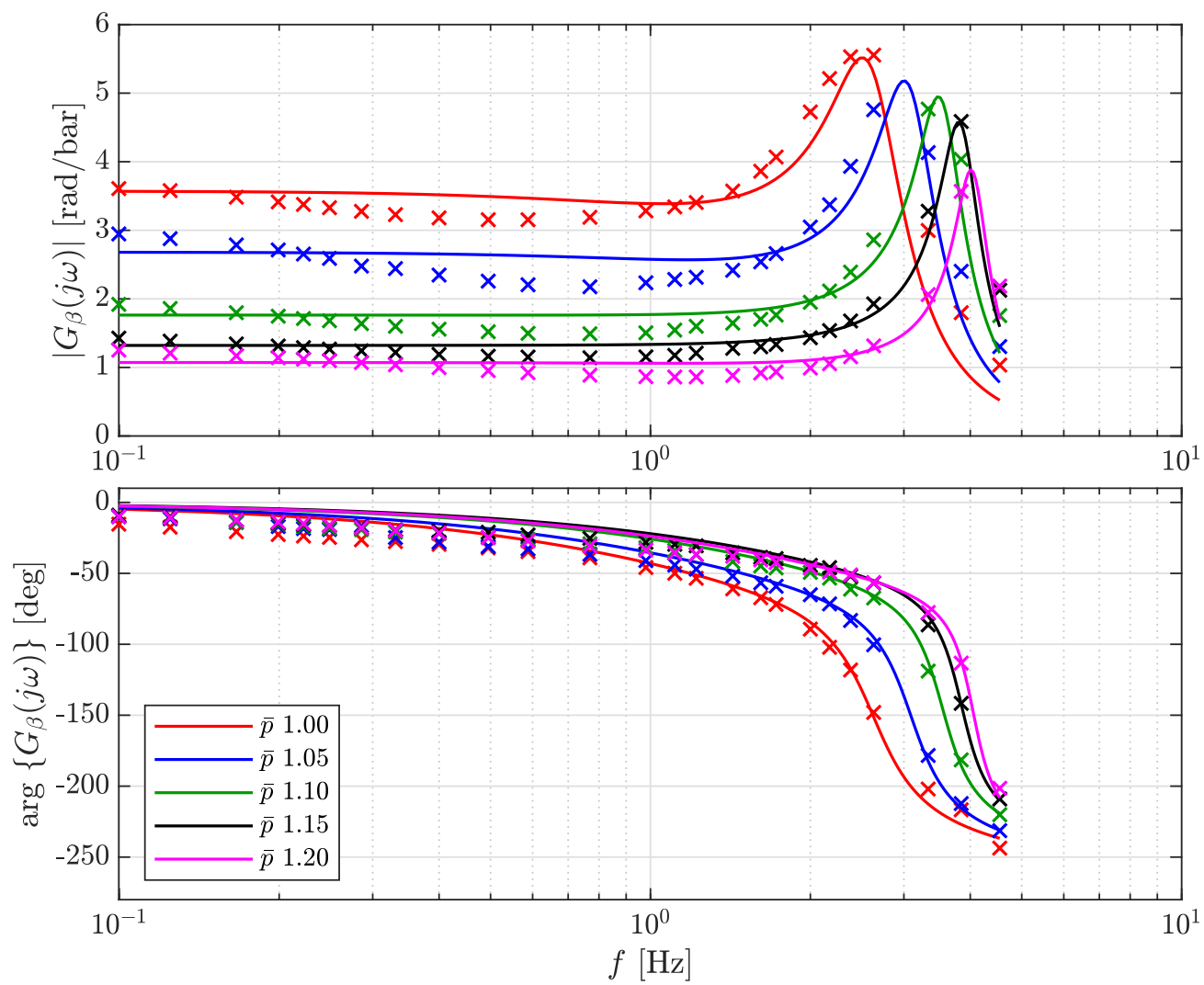

Figure A1. The frequency response in $\beta$-direction for different lower pressure levels $\bar{p}$ with the magnitude in the top plot and the phase in the bottom plot. The measured frequency responses resulting from the identification experiments are indicated by the crosses and the corresponding fits by the solid lines. 

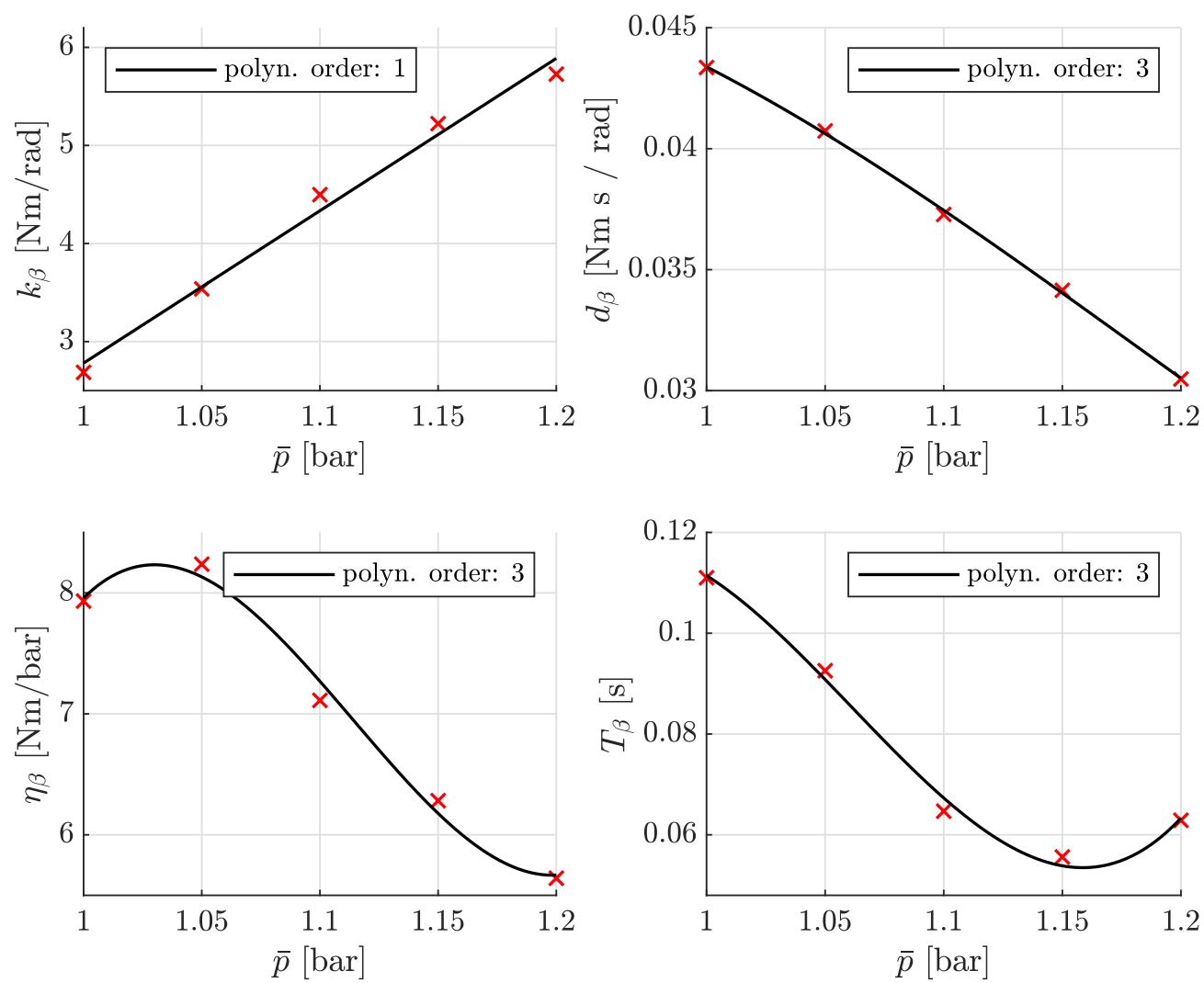

Figure A2. The parameters of the transfer function, $G_{\beta}(s, \bar{p}, m)$, as a function of the lower pressure level $\bar{p}$. The red crosses indicate the parameter values from the identification experiments and the black solid lines show the first or third order polynomial fits.

\section{References}

1. Magrini, E.; Flacco, F.; De Luca, A. Control of generalized contact motion and force in physical human-robot interaction. In Proceedings of the 2015 IEEE International Conference on Robotics and Automation (ICRA), Seattle, WA, USA, 26-30 May 2015; pp. 2298-2304. [CrossRef]

2. Ficuciello, F.; Villani, L.; Siciliano, B. Variable Impedance Control of Redundant Manipulators for Intuitive Human-Robot Physical Interaction. IEEE Trans. Robot. 2015, 31, 850-863. [CrossRef]

3. Li, X.; Pan, Y.; Chen, G.; Yu, H. Adaptive Human-Robot Interaction Control for Robots Driven by Series Elastic Actuators. IEEE Trans. Robot. 2017, 33, 169-182. [CrossRef]

4. Laschi, C.; Mazzolai, B.; Cianchetti, M. Soft robotics: Technologies and systems pushing the boundaries of robot abilities. Sci. Robot. 2016, 1, eaah3690. [CrossRef] [PubMed]

5. Polygerinos, P.; Correll, N.; Morin, S.A.; Mosadegh, B.; Onal, C.D.; Petersen, K.; Cianchetti, M.; Tolley, M.T.; Shepherd, R.F. Soft Robotics: Review of Fluid-Driven Intrinsically Soft Devices; Manufacturing, Sensing, Control, and Applications in Human-Robot Interaction. Adv. Eng. Mater. 2017, 19, 1700016. [CrossRef]

6. Sanan, S.; Ornstein, M.H.; Atkeson, C.G. Physical human interaction for an inflatable manipulator. In Proceedings of the Annual International Conference of the IEEE Engineering in Medicine and Biology Society, Boston, MA, USA, 30 August-3 September 2011. [CrossRef]

7. Abidi, H.; Cianchetti, M. On Intrinsic Safety of Soft Robots. Front. Robot. AI 2017, 4, 5. [CrossRef]

8. Rus, D.; Tolley, M. Design, fabrication and control of soft robots. Nature 2015, 521, 467-475. [CrossRef]

9. Walker, J.; Zidek, T.; Harbel, C.; Yoon, S.; Strickland, F.S.; Kumar, S.; Shin, M. Soft Robotics: A Review of Recent Developments of Pneumatic Soft Actuators. Actuators 2020, 9, 3. [CrossRef]

10. Gorissen, B.; Reynaerts, D.; Konishi, S.; Yoshida, K.; Kim, J.W.; De Volder, M. Elastic Inflatable Actuators for Soft Robotic Applications. Adv. Mater. 2017, 29, 1604977. [CrossRef]

11. Li, X.; Sun, K.; Guo, C.; Liu, T.; Liu, H. Design, modeling and characterization of a joint for inflatable robotic arms. Mechatronics 2020, 65, 102311. [CrossRef]

12. Yang, H.D.; Greczek, B.T.; Asbeck, A.T. Modeling and Analysis of a High-Displacement Pneumatic Artificial Muscle With Integrated Sensing. Front. Robot. AI 2019, 5, 136. [CrossRef] 
13. Gaiser, I.; Wiegand, R.; Ivlev, O.; Andres, A.; Breitwieser, H.; Schulz, S.; Bretthauer, G. Compliant Robotics and Automation with Flexible Fluidic Actuators and Inflatable Structures; InTech: New York, NY, USA, 2012. [CrossRef]

14. Niiyama, R.; Sun, X.; Sung, C.; An, B.; Rus, D.; Kim, S. Pouch Motors: Printable Soft Actuators Integrated with Computational Design. Soft Robot. 2015, 2, 59-70. [CrossRef]

15. Marchese, A.D.; Katzschmann, R.K.; Rus, D. A Recipe for Soft Fluidic Elastomer Robots. Soft Robot. 2015, 2, 7-25. [CrossRef] [PubMed]

16. Grzesiak, A.; Becker, R.; Verl, A. The Bionic Handling Assistant: A success story of additive manufacturing. Assem. Autom. 2011, 31, 329-333. [CrossRef]

17. Bodily, D.M.; Allen, T.F.; Killpack, M.D. Multi-objective design optimization of a soft, pneumatic robot. In Proceedings of the 2017 IEEE International Conference on Robotics and Automation (ICRA), Singapore, 29 May-3 June 2017; pp. 1864-1871. [CrossRef]

18. Yang, H.D.; Asbeck, A.T. A Layered Manufacturing Approach for Soft and Soft-Rigid Hybrid Robots. Soft Robot. 2020, 7, 218-232. [CrossRef] [PubMed]

19. Hofer, M.; Spannagl, L.; D'Andrea, R. Iterative Learning Control for Fast and Accurate Position Tracking with an Articulated Soft Robotic Arm. In Proceedings of the IEEE/RSJ International Conference on Intelligent Robots and Systems (IROS), Macao, China, 3-8 November 2019. [CrossRef]

20. Nguyen, P.H.; Zhang, W. Design and Computational Modeling of Fabric Soft Pneumatic Actuators for Wearable Assistive Devices. Nat. Sci. Rep. 2020, 10, 9638. [CrossRef] [PubMed]

21. Althoefer, K. Antagonistic actuation and stiffness control in soft inflatable robots. Nat. Rev. Mater. 2018, 3, 76-77. [CrossRef]

22. Gillespie, M.T.; Best, C.M.; Killpack, M.D. Simultaneous position and stiffness control for an inflatable soft robot. In Proceedings of the IEEE International Conference on Robotics and Automation (ICRA), Stockholm, Sweden, 16-24 May 2016. [CrossRef]

23. Hofer, M.; D'Andrea, R. Design, fabrication, modeling and control of a fabric-based spherical robotic arm. Mechatronics 2020, 68, 102369. [CrossRef]

24. Yang, H.D.; Asbeck, A.T. Design and Characterization of a Modular Hybrid Continuum Robotic Manipulator. IEEE/ASME Trans. Mechatron. 2020, 25, 2812-2823. [CrossRef]

25. Qiao, Z.; Nguyen, P.H.; Polygerinos, P.; Zhang, W. Dynamic Modeling and Motion Control of a Soft Robotic Arm Segment. In Proceedings of the 2019 American Control Conference (ACC), Philadelphia, PA, USA, 10-2 July 2019. [CrossRef]

26. Katzschmann, R.K.; Thieffry, M.; Goury, O.; Kruszewski, A.; Guerra, T.M.; Duriez, C.; Rus, D. Dynamically Closed-Loop Controlled Soft Robotic Arm using a Reduced Order Finite Element Model with State Observer. In Proceedings of the 2019 2nd IEEE International Conference on Soft Robotics (RoboSoft), Seoul Korea, 14-18 April 2019; pp. 717-724. [CrossRef]

27. Santina, C.D.; Katzschmann, R.K.; Bicchi, A.; Rus, D. Model-based dynamic feedback control of a planar soft robot: Trajectory tracking and interaction with the environment. The Int. J. Robot. Res. 2020, 39, 490-513. [CrossRef]

28. Marchese, A.D.; Katzschmann, R.K.; Rus, D. Whole arm planning for a soft and highly compliant 2D robotic manipulator. In Proceedings of the IEEE/RSJ International Conference on Intelligent Robots and Systems, Chicago, IL, USA, 14-18 September 2014; pp. 554-560. [CrossRef]

29. Jordan, M.; Pietrusky, D.; Mihajlov, M.; Ivlev, O. Precise position and trajectory control of pneumatic soft-actuators for assistance robots and motion therapy devices. In Proceedings of the IEEE International Conference on Rehabilitation Robotics, Kyoto, Japan, 23-26 June 2009; pp. 663-668. [CrossRef]

30. Gillespie, M.T.; Best, C.M.; Townsend, E.C.; Wingate, D.; Killpack, M.D. Learning nonlinear dynamic models of soft robots for model predictive control with neural networks. In Proceedings of the 2018 IEEE International Conference on Soft Robotics (RoboSoft), Livorno, Italy, 24-28 April 2018; pp. 39-45. [CrossRef]

31. Hyatt, P.; Johnson, C.C.; Killpack, M.D. Model Reference Predictive Adaptive Control for Large-Scale Soft Robots. Front. Robot. AI 2020, 7, 132. [CrossRef]

32. Ansari, Y.; Manti, M.; Falotico, E.; Cianchetti, M.; Laschi, C. Multiobjective Optimization for Stiffness and Position Control in a Soft Robot Arm Module. IEEE Robot. Autom. Lett. 2018, 3, 108-115. [CrossRef]

33. Mosadegh, B.; Polygerinos, P.; Keplinger, C.; Wennstedt, S.; Shepherd, R.F.; Gupta, U.; Shim, J.; Bertoldi, K.; Walsh, C.J.; Whitesides, G.M. Pneumatic networks for soft robotics that actuate rapidly. Adv. Funct. Mater. 2014, 24, 2163-2170. [CrossRef]

34. Della Santina, C.; Bianchi, M.; Grioli, G.; Angelini, F.; Catalano, M.; Garabini, M.; Bicchi, A. Controlling Soft Robots: Balancing Feedback and Feedforward Elements. IEEE Robot. Autom. Mag. 2017, 24, 75-83. [CrossRef]

35. Angelini, F.; Santina, C.D.; Garabini, M.; Bianchi, M.; Gasparri, G.M.; Grioli, G.; Catalano, M.G.; Bicchi, A. Decentralized Trajectory Tracking Control for Soft Robots Interacting With the Environment. IEEE Trans. Robot. 2018, 34, 924-935. [CrossRef]

36. Angelini, F.; Mengacci, R.; Santina, C.D.; Catalano, M.G.; Garabini, M.; Bicchi, A.; Grioli, G. Time Generalization of Trajectories Learned on Articulated Soft Robots. IEEE Robot. Autom. Lett. 2020, 5, 3493-3500. [CrossRef]

37. Katzschmann, R.K.; Marchese, A.D.; Rus, D. Autonomous object manipulation using a soft planar grasping manipulator. Soft Robot. 2015, 2, 155-164. [CrossRef]

38. Marchese, A.D.; Tedrake, R.; Rus, D. Dynamics and trajectory optimization for a soft spatial fluidic elastomer manipulator. Int. J. Robot. Res. 2016, 35, 1000-1019. [CrossRef]

39. Liang, X.; Cheong, H.; Sun, Y.; Guo, J.; Chui, C.K.; Yeow, C.H. Design, Characterization, and Implementation of a Two-DOF Fabric-Based Soft Robotic Arm. IEEE Robot. Autom. Lett. 2018, 3, 2702-2709. [CrossRef] 
40. Hyatt, P.; Kraus, D.; Sherrod, V.; Rupert, L.; Day, N.; Killpack, M.D. Configuration Estimation for Accurate Position Control of Large-Scale Soft Robots. IEEE/ASME Trans. Mechatron. 2019, 24, 88-99. [CrossRef]

41. Das, A.; Nabi, M. A review on Soft Robotics: Modeling, Control and Applications in Human-Robot interaction. In Proceedings of the 2019 International Conference on Computing, Communication, and Intelligent Systems (ICCCIS), Greater Noida, India, 18-19 October 2019; pp. 306-311. [CrossRef]

42. Liang, X.; Cheong, H.; Chui, C.K.; Yeow, C.H. A Fabric-Based Wearable Soft Robotic Limb. ASME. J. Mech. Robot. 2019, 11, 031003. [CrossRef]

43. Nguyen, P.H.; Sparks, C.; Nuthi, S.G.; Vale, N.M.; Polygerinos, P. Soft Poly-Limbs: Toward a New Paradigm of Mobile Manipulation for Daily Living Tasks. Soft Robot. 2019, 6, 38-53. [CrossRef]

44. Thalman, C.M.; Lam, Q.P.; Nguyen, P.H.; Sridar, S.; Polygerinos, P. A Novel Soft Elbow Exosuit to Supplement Bicep Lifting Capacity. In Proceedings of the 2018 IEEE/RSJ International Conference on Intelligent Robots and Systems (IROS), Madrid, Spain, 1-5 October 2018; pp. 6965-6971. [CrossRef]

45. Polygerinos, P.; Wang, Z.; Galloway, K.C.; Wood, R.J.; Walsh, C.J. Soft robotic glove for combined assistance and at-home rehabilitation. Robot. Auton. Syst. 2015, 73, 135-143. [CrossRef]

46. Qi, R.; Khajepour, A.; Melek, W.W.; Lam, T.L.; Xu, Y. Design, Kinematics, and Control of a Multijoint Soft Inflatable Arm for Human-Safe Interaction. IEEE Trans. Robot. 2017, 33, 594-609. [CrossRef]

47. Santina, C.D.; Truby, R.L.; Rus, D. Data-Driven Disturbance Observers for Estimating External Forces on Soft Robots. IEEE Robot. Autom. Lett. 2020, 5, 5717-5724. [CrossRef]

48. Zughaibi, J.; Hofer, M.; D'Andrea, R. A Fast and Reliable Pick-and-Place Application with a Spherical Soft Robotic Arm. In Proceedings of the 2021 IEEE 4th International Conference on Soft Robotics (RoboSoft), New Haven, CT, USA, 12-16 April 2021; pp. 599-606. [CrossRef]

49. Robots and Robotic Devices-Collaborative Robots. Standard, International Organization for Standardization. Ann. Lab. Med. 2017, 37, 365-370. [CrossRef] [PubMed]

50. Stronge, W.J. Impact Mechanics, 2nd ed.; Cambridge University Press: Cambridge, UK, 2018. [CrossRef]

51. Haddadin, S.; Albu-Schäffer, A.; Hirzinger, G. Requirements for Safe Robots: Measurements, Analysis and New Insights. Int. J. Robot. Res. 2009, 28, 1507-1527. [CrossRef]

52. Hofer, M.; Sferrazza, C.; D'Andrea, R. A Vision-Based Sensing Approach for a Spherical Soft Robotic Arm. Front. Robot. AI 2021, 8, 8. [CrossRef] [PubMed]

53. Troughton, M.J. Handbook of Plastics Joining: A Practical Guide (Plastics Design Library), 2nd ed.; William Andrew: Norwich, NY, USA, 2008.

54. Zughaibi, J.; Hofer, M.; D'Andrea, R. A Fast and Reliable Pick-and-Place Application with a Spherical Soft Robotic Arm. Online appendix. 2021. [CrossRef]

55. Ljung, L. System Identification: Theory for the User, 2nd ed.; Prentice Hall PTR: Upper Saddle River, NJ, USA, 1999.

56. Briat, C. Linear parameter-varying and time-delay systems. Anal. Obs. Filter. Control 2014, 3, 5-7.

57. Bacciotti, A. Stability and Control of Linear Systems, 1st ed.; Springer: Cham, Switzerland, 2019. [CrossRef] 